\title{
20. MAJOR AND TRACE ELEMENT GEOCHEMISTRY OF SEDIMENTS FROM DEEP SEA DRILLING PROJECT, LEG 27, SITES 259-263, EASTERN INDIAN OCEAN ${ }^{1}$
}

\author{
Peter J. Cook, Bureau of Mineral Resources, Canberra, Australia
}

\begin{abstract}
A total of 56 samples from five sites in the eastern Indian Ocean was analyzed for a wide range of major and trace elements. The sediments may be divided into clay, which is low in $\mathrm{CaCO}_{3}$ and predominantly of Mesozoic age, and calcareous ooze, which is high in $\mathrm{CaCO}_{3}$ and predominantly of Cenozoic age. Both groups of sediments have lower trace element contents than pelagic sediments in other oceans.

Statistical treatment of the data made it possible to divide the oxides and elements into a number of mineralogically significant groups. These include the aluminosilicate group, the biogenic carbonate group, the biogenic silica group, and the ferromanganese group. Some variables (such as $\mathrm{Fe}_{2} \mathrm{O}_{3}$ ) are in more than one group, and a few could not be assigned to any group.
\end{abstract}

\section{INTRODUCTION}

In order to more clearly understand the nature and origin of the sediments encountered during the course of deep-sea drilling in the eastern Indian Ocean, the writer obtained a number of major and trace element analyses on Leg 27 sediments. Where possible, at least two samples were obtained from every sedimentary unit; within each unit the samples were chosen randomly. Objects of this geochemical study include: (1) the chemical characterization of the sediments; (2) the determination as to whether groups of sediments from different eras, depositional environments, or geographic locations are in any way geochemically different; and (3) the interrelations of elements or groups of elements.

The geographic locations of the drill sites from which the samples were obtained are shown in Figure 1. The vertical distribution of samples at Sites $259-263$ is indicated in Figure 2.

In addition to chemical analyses, most of the samples were analyzed by X-ray diffraction in order to relate geochemistry to mineralogy. A number of impregnated thin sections were also prepared to obtain further mineralogical information on the samples.

\section{ANALYTICAL TECHNIQUES}

All major and trace element analyses were undertaken by the Analytical Chemistry Section of the Australian Mineral Development Laboratories.

A rapid silicate analysis method similar to that described by Bowditch et al. (1969) was used for $\mathrm{SiO}_{2}$, $\mathrm{Al}_{2} \mathrm{O}_{3}, \mathrm{Fe}_{2} \mathrm{O}_{3}, \mathrm{CaO}, \mathrm{MgO}, \mathrm{Na}_{2} \mathrm{O}, \mathrm{K}_{2} \mathrm{O}, \mathrm{MnO}, \mathrm{P}_{2} \mathrm{O}$,

\footnotetext{
'Published with the permission of the Director, Bureau of Mineral Resources, Geology and Geophysics, Canberra, Australia.
}

$\mathrm{TiO}_{2}, \mathrm{Cr}_{2} \mathrm{O}_{3}$, and $\mathrm{V}_{2} \mathrm{O}_{5}$. A Jarrell-Ash direct-reading emission spectrometer was used for the analyses.

$\mathrm{CO}_{2}$ was obtained by gaseous evolution after attack by boiling acid, then absorbtion of $\mathrm{CO}_{2}$, and subsequent gravimetric determination. Total $\mathrm{H}_{2} \mathrm{O}$ was determined by the classical gravimetric Penfold technique.

$\mathrm{Sr}, \mathrm{Ba}, \mathrm{Li}, \mathrm{Cu}, \mathrm{Pb}, \mathrm{Zn}, \mathrm{Co}, \mathrm{Ni}$, and $\mathrm{Cr}$ were determined by atomic absorption spectrometry on total solutions of the samples using mixed acids and HF.

$\mathrm{X}$-ray diffraction analysis was carried out by the Australian Bureau of Mineral Resources using a Phillips $\mathrm{X}$-ray unit with a stabilized power supply. A spinner was used to overcome any preferred orientation effects. Results were not quantified, and analyses were generally on the total sediment. In a few instances the $<2 \mu$ fraction was separated and subjected to X-ray analysis.

\section{RESULTS}

A total of 56 samples was analyzed; a number of analyses were repeated in order to evaluate the level of precision. Results are given in Tables 1-5. Average values are given in Tables 6-8. Good levels of precision were obtained for $\mathrm{SiO}_{2}( \pm 1.25 \%), \mathrm{Al}_{2} \mathrm{O}_{3}( \pm 1.39 \%)$, $\mathrm{Fe}_{2} \mathrm{O}_{3}( \pm 2.54 \%) \mathrm{CaO}( \pm 0.40 \%), \mathrm{MgO}( \pm 1.85 \%), \mathrm{Na}_{2} \mathrm{O}$ $( \pm 3.11 \%), \mathrm{TiO}_{2}( \pm 0.40 \%)$, loss on ignition (LOI) $( \pm 0.22 \%), \mathrm{CO}_{2}( \pm 1.05 \%)$, and $\mathrm{Cu}( \pm 2.78 \%)$. Moderate precision was obtained for $\mathrm{K}_{2} \mathrm{O}( \pm 9.51 \%), \mathrm{Mn}$ $( \pm 5.28 \%), \mathrm{H}_{2} \mathrm{O}( \pm 7.45 \%)$, and $\mathrm{Zn}( \pm 6.59 \%)$. Poor levels of precision were obtained for $\mathrm{P}_{2} \mathrm{O}_{3}( \pm 15.47 \%), \mathrm{Pb}$ $( \pm 30.0 \%)$, $\mathrm{Co}( \pm 11.90 \%)$, and $\mathrm{Ni}( \pm 25.9 \%) . \mathrm{Cr}_{2} \mathrm{O}_{3}$ and $\mathrm{V}_{2} \mathrm{O}_{5}$ contents were in general below the level of detection.

Bowditch et al. (1969) claim good accuracy for analyses by direct-reading emission spectroscopy. Comparison of Leg 27 results (Table 6) with those of El Wakeel and Riley (1961) suggests acceptable accuracy for the major oxides $\left(\mathrm{SiO}_{2}, \mathrm{Al}_{2} \mathrm{O}_{3}\right.$, etc. $)$. There are major 


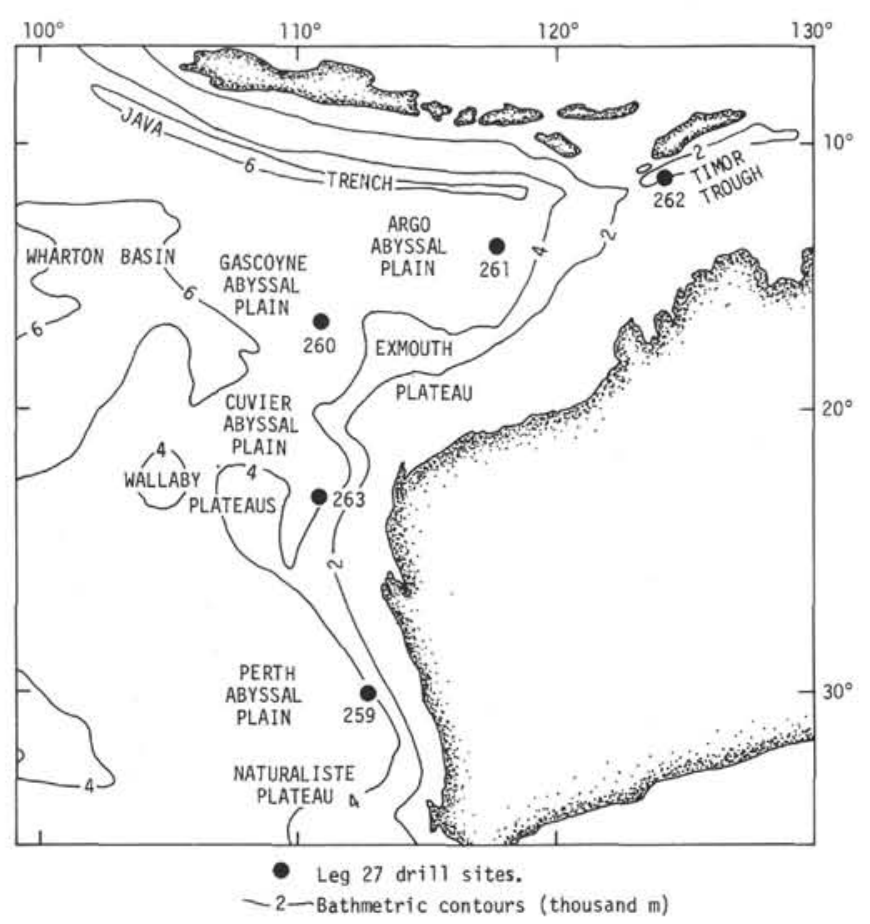

Figure 1. Locality map indicating Leg 27 drilling sites.

differences between mean trace element values for Leg 27 sediments and those obtained by Goldberg and Arrhenius (1958) for Pacific pelagic sediments (Table 8). The Leg 27 values are generally lower than the Pacific values.

Comparison of Leg 27 values with those reported by Turekian and Wedepohl (1961) also suggests that the Indian Ocean material tends to be somewhat impoverished in $\mathrm{Cu}, \mathrm{Pb}, \mathrm{Zn}, \mathrm{Co}$, and $\mathrm{Ni}$ compared with the average values for pelagic sediments. The reason for this may be that much of the Mesozoic sedimentary sequence is not pelagic. This is undoubtedly supported by the low $\mathrm{Mn}, \mathrm{Co}$, and $\mathrm{Ni}$ values of the Leg 27 Mesozoic sediments.

Using a program developed by $\mathrm{W}$. Mayo, of the Bureau of Mineral Resources, Canberra, $X-Y$ plots and summary statistics were obtained for all variables. Correlation lines were determined by the least-squares regression method, and correlation coefficients for the sediments as a whole are given in Table 9. These coefficients are tested against two limits. The first assumes a population correlation coefficient of zero $(\rho=0)$ and a $99 \%$ confidence level $(\alpha=0.01)$. At this confidence level the correlation coefficient must be greater than +0.341 or less than -0.341 .

Possibly more acceptable limits are imposed by taking the values used by Chave and Mackenzie (1961) of a population correlation coefficient $\rho$ of greater than +0.80 and less thani- 0.80 at level of significance of $\alpha=$ 0.05 . This gives a limit for acceptability of \pm 0.680 .

Because the limiting value of the correlation coefficient varies with the size of the sample population, other limits are given where the samples are split into several populations (Table 9-13).

\section{DISCUSSION}

The summarized geochemical results (Tables 6 and 7) indicate that there are marked differences in the composition of the Mesozoic and Cenozoic sediments. This is supported by the X-ray diffraction data of the writer and of Cook, Zemmels, and Matti.

The X-ray data show that the Cenozoic sediments are composed predominantly of calcite, with varying amounts of quartz and minor to trace quantities of clay minerals and zeolites. Mesozoic sediments, on the other hand, are generally composed of abundant quartz and clay minerals, with varying amounts of zeolites and minor to trace quantities of calcite. The fundamental difference, then, is in the abundance of carbonate. This difference is apparent from Figure 3 in which the abundance of $\mathrm{CaO}$ (occurring almost exclusively as $\mathrm{CaCO}_{3}$ or, rarely, as $[\mathrm{Ca}, \mathrm{Mg}] \mathrm{CO}_{3}$ ) is seen to have a polymodal distribution. Mesozoic sediments generally contain $10 \%$ or less of $\mathrm{CaO}$, and Cenozoic sediments predominantly contain more than $10 \% \mathrm{CaO}$. It should nevertheless be noted that there are some carbonate-rich sediments of Mesozoic age, and conversely some carbonate-poor sediments of Cenozoic age. Therefore, sediments recovered during Leg 27 drilling may be divided into two similar groups either according to age (Mesozoic and Cenozoic) or according to carbonate abundance (clay and calcareous ooze).

If Mesozoic and Cenozoic sediments are considered on a carbonate-free, water-free basis (Table 7), the recalculated major oxides are somewhat similar in both groups, although the Mesozoic sediments are more siliceous and the Cenozoic sediments more aluminous. When the recalculated values of Leg 27 material (Table 7) are compared with those of El Wakeel and Riley (1961), the Leg 27 Mesozoic sediments closely resemble the average siliceous sediment, whereas the Cenozoic sediments are similar both to the average argillaceous and calcareous pelagic sediments of El Wakeel and Riley (1961).

If trace element contents of sediments are considered (Table 8), the Leg 27 values are in general below those reported by Goldberg and Arrhenius (1958) for Pacific pelagic sediments except strontium and barium which are higher, possibly due to the greater carbonate abundance. If the composition of Mesozoic sediments is compared with the average trace element content of pelagic clays (Turekian and Wedepohl, 1961), it is apparent (Table 8) that the Mesozoic sediments are lower in all trace elements (except strontium and lithium) than the average pelagic clay. There are probably various factors responsible for these differences. Significant portions of the Mesozoic sequence are probably not pelagic; closeness of the sites to the continental margin apparently resulted in a comparatively high rate of terrigenous sedimentation (resulting in a dilution of chemical sediments). In addition, the average values of Turekian and Wedepohl are weighted by the high Pacific Ocean values.

If the average values for Cenozoic sediments are compared with those given by Turekian and Wedepohl (1961), the Leg 27 material has lower strontium and 

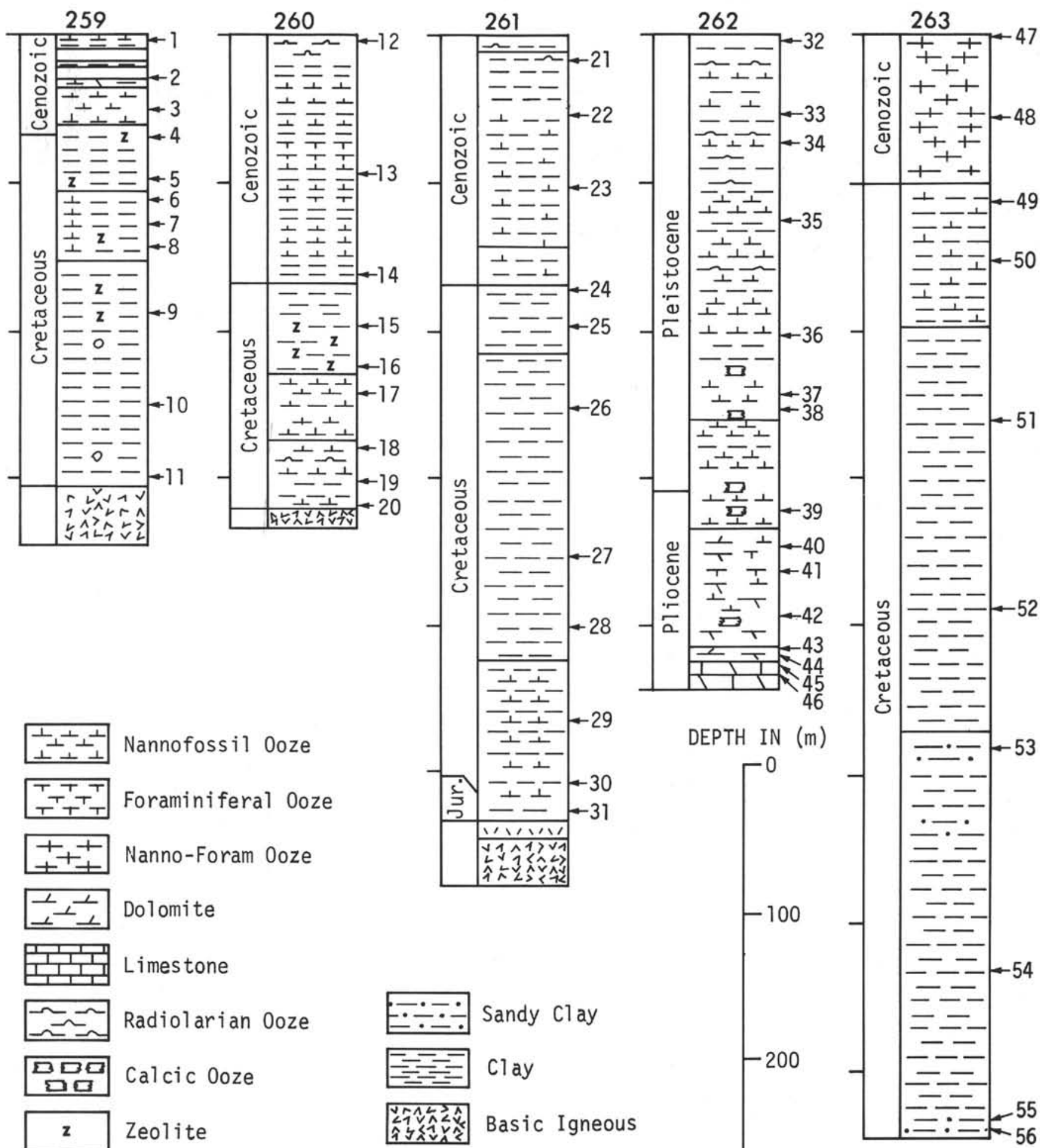

$\pm \perp+1$ Dolomite

Limestone

Radiolarian Ooze
zalcic Ooze
(a) Meolite
Manganese Nodule

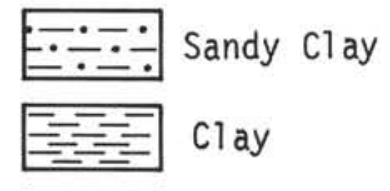

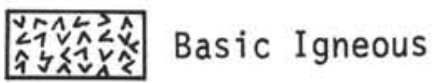

+29 Sample location

and number

(as in Tables 1-5)

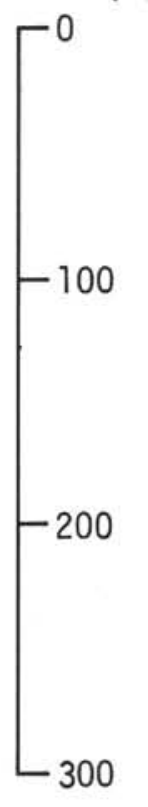

56

Figure 2. Generalized stratigraphic columns of the five DSDP sites drilled on Leg 27. Sampling positions are indicated. 
TABLE 1

Major and Trace Element Analyses, Site 259

\begin{tabular}{|c|c|c|c|c|c|c|c|c|c|c|c|}
\hline \multirow[b]{2}{*}{ Element } & \multicolumn{11}{|c|}{ Sample } \\
\hline & 1 & 2 & 3 & 4 & 5 & 6 & 7 & 8 & 9 & 10 & 11 \\
\hline \multicolumn{12}{|l|}{ Major } \\
\hline $\mathrm{SiO}_{2}{ }^{\mathrm{a}}$ & 14.5 & 48.5 & 19.4 & 58.5 & 55.0 & 51.4 & 43.5 & 33.0 & 59.0 & 61.0 & 62.0 \\
\hline $\mathrm{A}_{2} \mathrm{O}_{3}$ & 2.0 & 9.4 & 3.6 & 11.3 & 10.8 & 10.4 & 8.9 & 7.5 & 14.4 & 14.3 & 11.7 \\
\hline $\mathrm{Fe}_{2} \mathrm{O}_{3}$ & 1.1 & 5.0 & 1.7 & 6.3 & 5.6 & 5.0 & 4.4 & 3.2 & 5.1 & 6.4 & 6.0 \\
\hline $\mathrm{CaO}$ & 40.0 & 10.1 & 34.0 & 1.1 & 5.2 & 9.2 & 15.0 & 24.1 & 1.25 & 1.05 & 1.1 \\
\hline $\mathrm{MgO}$ & 1.15 & 1.9 & 1.1 & 2.3 & 2.1 & 2.0 & 1.9 & 1.4 & 2.1 & 2.2 & 2.4 \\
\hline $\mathrm{Na}_{2} \mathrm{O}$ & 1.85 & 3.1 & 2.35 & 3.15 & 3.15 & 3.05 & 2.65 & 2.15 & 2.7 & 2.35 & 2.9 \\
\hline $\mathrm{K}_{2} \mathrm{O}$ & 0.50 & 2.65 & 1.1 & 3.0 & 2.8 & 2.7 & 2.1 & 1.5 & 2.1 & 2.1 & 2.0 \\
\hline MnO & $<0.02$ & 0.24 & $<0.02$ & 0.65 & 0.31 & 0.27 & 0.29 & 0.28 & 0.04 & 0.04 & 0.09 \\
\hline $\mathrm{P}_{2} \mathrm{O}_{5}$ & 0.04 & 0.25 & 0.07 & 0.29 & 0.04 & 0.02 & 0.02 & $<0.02$ & 0.05 & 0.10 & 0.14 \\
\hline $\mathrm{TiO}_{2}$ & 0.11 & 0.25 & 0.13 & 0.49 & 0.41 & 0.41 & 0.36 & 0.28 & 0.49 & 0.61 & 0.55 \\
\hline $\mathrm{Cr}_{2} \mathrm{O}_{3}$ & $<0.1$ & 0.34 & $<0.1$ & $<0.1$ & $<.01$ & $<0.1$ & $<0.1$ & $<0.1$ & $<0.1$ & $<0.1$ & $<0.1$ \\
\hline $\mathrm{v}_{2} \mathrm{O}_{5}$ & $<0.05$ & $<0.05$ & $<0.05$ & $<0.05$ & $<0.05$ & $<0.05$ & $<0.05$ & $<0.05$ & $<0.05$ & $<0.05$ & $<0.05$ \\
\hline LOIb & 38.2 & 18.6 & 36.6 & 12.9 & 14.8 & 15.4 & 20.9 & 26.3 & 12.4 & 9.5 & 11.4 \\
\hline Total & 100.3 & 100.3 & 100.1 & 99.9 & 100.2 & 99.8 & 100.0 & 99.7 & 99.6 & 99.6 & 100.2 \\
\hline \multicolumn{12}{|l|}{ Trace } \\
\hline $\mathrm{CO}_{2}{ }^{\mathrm{c}}$ & 32.0 & 6.4 & 27.4 & 0.15 & 3.6 & 6.4 & 10.5 & 18.4 & 0.25 & 0.3 & 0.6 \\
\hline $\mathrm{H}_{2} \mathrm{O}$ & 3.4 & 10.5 & 4.4 & 12.1 & 10.7 & 8.0 & 8.5 & 6.5 & 11.8 & 8.4 & 9.5 \\
\hline $\mathrm{Sr}$ & 0.11 & 0.027 & 0.07 & 0.015 & 0.015 & 0.021 & 0.031 & 0.036 & 0.008 & 0.009 & 0.018 \\
\hline $\mathrm{Ba}$ & 0.045 & 0.07 & 0.02 & 0.28 & 0.04 & 0.095 & 0.082 & 0.06 & 0.105 & 0.15 & 0.082 \\
\hline $\mathrm{Li}$ & 0.001 & 0.002 & 0.002 & 0.002 & 0.003 & 0.002 & 0.003 & 0.002 & 0.003 & 0.003 & 0.002 \\
\hline $\mathrm{Cu}$ & 0.0035 & 0.011 & 0.0025 & 0.011 & 0.0095 & 0.0075 & 0.0065 & 0.005 & 0.005 & 0.0065 & 0.006 \\
\hline $\mathrm{Pb}$ & 0.001 & 0.003 & 0.002 & 0.0015 & 0.0015 & 0.001 & 0.0015 & 0.0018 & 0.003 & 0.003 & 0.002 \\
\hline $\mathrm{Zn}$ & 0.0035 & 0.013 & 0.004 & 0.0105 & 0.011 & 0.0125 & 0.008 & 0.0095 & 0.0095 & 0.016 & 0.018 \\
\hline Co & $<0.0001$ & 0.0013 & 0.0002 & 0.0035 & 0.0008 & 0.0013 & 0.0008 & 0.0005 & 0.0015 & 0.004 & 0.002 \\
\hline $\mathrm{Ni}$ & 0.0025 & 0.006 & 0.0025 & 0.007 & 0.006 & 0.0075 & 0.0058 & 0.0045 & 0.004 & 0.0095 & 0.007 \\
\hline
\end{tabular}

Note: Each sample has the following properties:

(1) depth, $1.80 \mathrm{~m}$, Unit 1, Pleistocene, foram-bearing nanno ooze; (2) depth, 31.8m, Unit 1, lower Eocene. zeolite clay; (3) depth, 50.8m, Unit 1, upper Paleocene, nanno ooze, (4) depth, 69.8m, Unit 2, ?Lower Cretaceous, zeolite clay; (5) depth, 99.8m, Unit 2, Lower Cretaceous, zeolite clay; (6) depth, $112.8 \mathrm{~m}$, Unit 3, Lower Cretaceous, zeolite-rich nanno clay; (7) depth, $131.8 \mathrm{~m}$, Unit 3, Lower Cretaceous, zeolite-rich clayey nanno ooze; (8) depth, $145.8 \mathrm{~m}$, Unit 3, Lower Cretaceous, clay and zeolite-rich nanno ooze; (9) depth, 191.8m, Unit 4, Lower Cretaceous, quartz-bearing clay; (10) depth, 256.8m, Unit 4, Lower Cretaceous, zeolite-bearing clay; (11) depth, $300.8 \mathrm{~m}$, Unit 4, Lower Cretaceous, clay.

${ }^{a}$ All major and trace elements expressed as percentages. Analyses by Australian Mineral Development Laboratories.

$\mathrm{b}_{\mathrm{LOI}}=$ loss on ignition

${ }^{c}$ Components $\mathrm{Al}_{2} \mathrm{O}_{3}$ to $\mathrm{V}_{2} \mathrm{O}_{5}$ determined by direct-reading emission spectrography. $\mathrm{CO}_{2}$ and $\mathrm{H}_{2} \mathrm{O}$ by classical wet chemistry. Elements $\mathrm{Sr}$, $\mathrm{Ba}$, Li, $\mathrm{Cu}$, $\mathrm{Pb}, \mathrm{Zn}, \mathrm{Co}$, and $\mathrm{Ni}$ by atomic absorption spectroscopy. 
TABLE 2

Major and Trace Element Analyses, Site 260

\begin{tabular}{|c|c|c|c|c|c|c|c|c|c|}
\hline \multirow[b]{2}{*}{ Element } & \multicolumn{9}{|c|}{ Sample } \\
\hline & 12 & 13 & 14 & 15 & 16 & 17 & 18 & 19 & 20 \\
\hline \multicolumn{10}{|l|}{ Major } \\
\hline $\mathrm{SiO}_{2}$ & 14.0 & 47.5 & 48.4 & 57.5 & 55.5 & 18.2 & 77.0 & 77.0 & 48.4 \\
\hline $\mathrm{Al}_{2} \mathrm{O}_{3}$ & 3.2 & 16.2 & 15.6 & 12.1 & 12.5 & 3.3 & 4.5 & 5.0 & 2.2 \\
\hline $\mathrm{Fe}_{2} \mathrm{O}_{3}$ & 1.6 & 7.0 & 8.3 & 7.3 & 8.0 & 2.4 & 3.4 & 3.6 & 7.0 \\
\hline $\mathrm{CaO}$ & 38.0 & 1.8 & 2.8 & 0.90 & 1.0 & 38.0 & 1.8 & 1.8 & 17.5 \\
\hline $\mathrm{MgO}$ & 0.9 & 4.1 & 3.1 & 2.65 & 3.0 & 1.3 & 1.6 & 1.9 & 1.8 \\
\hline $\mathrm{Na}_{2} \mathrm{O}$ & 2.45 & 2.65 & 2.55 & 2.9 & 3.0 & 1.15 & 2.0 & 1.75 & 1.7 \\
\hline $\mathrm{K}_{2} \mathrm{O}$ & 0.55 & 2.1 & 3.4 & 3.0 & 3.3 & 0.6 & 1.7 & 2.1 & 1.9 \\
\hline $\mathrm{MnO}$ & 0.11 & 1.3 & 0.43 & 0.39 & 0.28 & 0.57 & 0.32 & 0.09 & 0.33 \\
\hline $\mathrm{P}_{2} \mathrm{O}_{5}$ & 0.09 & 0.57 & 0.29 & 0.09 & 0.1 & 0.06 & 0.08 & 0.12 & 0.18 \\
\hline $\mathrm{TiO}_{2}$ & 0.14 & 0.73 & 0.75 & 0.81 & 0.78 & 0.19 & 0.26 & 0.29 & 0.23 \\
\hline $\mathrm{Cr}_{2} \mathrm{O}_{3}$ & $<0.1$ & $<0.1$ & $<0.1$ & $<0.1$ & $<0.1$ & $<0.1$ & $<0.1$ & 0.09 & $<0.1$ \\
\hline $\mathrm{v}_{2} \mathrm{O}_{5}$ & $<0.05$ & $<0.05$ & $<0.05$ & $<0.05$ & $<0.05$ & $<0.05$ & $<0.05$ & $<0.05$ & $<0.05$ \\
\hline LOI & 39.2 & 15.7 & 14.2 & 12.2 & 12.2 & 34.3 & 7.3 & 6.2 & 18.1 \\
\hline Total & 100.2 & 99.6 & 99.8 & 99.8 & 99.7 & 100.1 & 100.0 & 100.21 & 99.3 \\
\hline \multicolumn{10}{|l|}{ Trace } \\
\hline $\mathrm{CO}_{2}$ & 31.0 & 0.3 & 0.4 & 0.2 & 0.15 & 30.5 & 1.1 & 0.9 & 12.7 \\
\hline $\mathrm{H}_{2} \mathrm{O}$ & 3.8 & 14.7 & 12.2 & 11.5 & 11.6 & 3.6 & 6.2 & 5.6 & 4.4 \\
\hline $\mathrm{Sr}$ & 0.094 & 0.012 & 0.012 & 0.015 & 0.013 & 0.024 & 0.009 & 0.008 & 0.037 \\
\hline $\mathrm{Ba}$ & 0.046 & 0.07 & 0.04 & 0.025 & 0.035 & 0.07 & 0.025 & 0.023 & 0.012 \\
\hline $\mathrm{Li}$ & 0.001 & 0.007 & 0.005 & 0.003 & 0.003 & 0.002 & 0.005 & 0.005 & 0.002 \\
\hline $\mathrm{Cr}$ & 0.0065 & 0.03 & 0.095 & 0.0105 & 0.095 & 0.065 & 0.016 & 0.0045 & 0.02 \\
\hline $\mathrm{Pb}$ & 0.001 & 0.001 & 0.0018 & 0.0015 & 0.002 & 0.001 & 0.0001 & 0.001 & 0.0013 \\
\hline $\mathrm{Zn}$ & 0.005 & 0.016 & 0.017 & 0.0115 & 0.0125 & 0.0065 & 0.006 & 0.008 & 8.014 \\
\hline Co & 0.0001 & 0.0085 & 0.0045 & 0.0035 & 0.002 & 0.001 & 0.0008 & 0.001 & 0.0013 \\
\hline $\mathrm{Ni}$ & 0.004 & 0.032 & 0.0115 & 0.007 & 0.007 & 0.004 & 0.003 & 0.0045 & 0.01 \\
\hline
\end{tabular}

Note: Each sample has the following properties:

(12) depth, 0.3m, Unit 1, Pleistocene, radiolarian ooze; (13) depth, $96.3 \mathrm{~m}$, Unit 1, middle Miocene or younger, clay and nanno clay; (14) depth, $165.8 \mathrm{~m}$, Unit 2, upper Paleocene or younger, nanno-bearing clay; (15) depth, 200.8m, Unit 2, Lower Cretaceous, zeolite-rich clay; (16) depth, 222.8m, Unit 2, Lower Cretaceous, zeolite-bearing clay; (17) depth, 245.4m, Unit 3, Lower Cretaceous, nanno ooze; (18) depth, 282.7m, Unit 4, Lower Cretaceous, zeolite and nanno-rich clay; (19) depth, $301.2 \mathrm{~m}$, Unit 4, Lower Cretaceous, clay; (20) depth, 320.6m, Unit 4, Lower Cretaceous, nanno ooze.

copper contents, but, surprisingly, higher barium, lead, zinc, cobalt, and nickel contents than the oceanic average. If this feature is further examined, it is found that the abyssal zone Cenozoic sediments of Leg 27 have higher trace element contents than the shallower Cenozoic sediments of the Timor Trough. This suggests that a possible explanation for the high Leg 27 values is that the carbonates encountered at Sites 259, 260, 261, and 263 are all in deep water, below the lysocline, and are considerably deeper than most of the calcareous oozes used by Turekian and Wedepohl (1961). Since deep abyssal ocean waters tend to be richer in trace elements than shallower water, this difference may be reflected in the sediments, as a consequence of adsorption of cations by the sediments.
In general, the bulk chemistry of Mesozoic sediments at all sites (except 262) is similar (Tables 1-5); the main difference is the abundance of $\mathrm{CaCO}_{3}$. The Cenozoic sediments of Site 262 also differ from the other four sites in the abundance of $\mathrm{CaCO}_{3}$, and, in addition, the $\mathrm{MgO}$ content is much higher at Site 262 because of the abundance of dolomite rhombs. This feature is discussed by the writer in another paper in this volume.

The masking effect of calcareous material is also evident when the analyses are statistically treated. The matrix of correlation coefficients for all samples (Table 9), is masked by the closed array effect, which in these samples is dominated by calcium carbonate. The problems of dealing with a group of variables with a constant $100 \%$ summation is considered in some detail 
TABLE 3

Major and Trace Element Analyses, Site 261

\begin{tabular}{|c|c|c|c|c|c|c|c|c|c|c|c|}
\hline Element & 21 & 22 & 23 & 24 & 25 & 26 & 27 & 28 & 29 & 30 & 31 \\
\hline $\mathrm{SiO}_{2}$ & 56.0 & 19.3 & 14.2 & 52.0 & 54.5 & 77.0 & 71.5 & 81.4 & 71.5 & 61.0 & 15.8 \\
\hline $\mathrm{Al}_{2} \mathrm{O}_{3}$ & 14.1 & 6.5 & 4.7 & 15.4 & 13.2 & 4.8 & 7.1 & 4.2 & 6.7 & 11.5 & 2.9 \\
\hline $\mathrm{Fe}_{2} \mathrm{O}_{3}$ & 4.3 & 3.0 & 1.8 & 8.3 & 7.9 & 5.0 & 5.6 & 3.6 & 5.7 & 6.3 & 3.1 \\
\hline $\mathrm{Na}_{2} \mathrm{O}$ & 4.8 & 1.7 & 1.4 & 2.4 & 3.05 & 1.85 & 2.3 & 1.35 & 1.55 & 2.0 & 0.66 \\
\hline $\mathrm{K}_{2} \mathrm{O}$ & 2.2 & 1.2 & 0.7 & 3.3 & 3.1 & 1.3 & 2.3 & 1.4 & 1.5 & 2.1 & 0.7 \\
\hline $\mathrm{MnO}$ & 1.2 & 0.18 & 0.08 & 0.77 & 1.44 & 0.04 & 0.05 & 0.03 & 0.49 & 0.55 & 0.54 \\
\hline $\mathrm{P}_{2} \mathrm{O}_{5}$ & 0.14 & 0.08 & 0.05 & 0.56 & 0.08 & 0.01 & 0.06 & $<0.03$ & 0.14 & 0.27 & 0.03 \\
\hline $\mathrm{TiO}_{2}$ & 0.55 & 0.25 & 0.19 & 0.89 & 0.67 & 0.34 & 0.57 & 0.27 & 0.39 & 0.49 & 0.2 \\
\hline \multicolumn{12}{|l|}{ Trace } \\
\hline $\mathrm{CO}_{2}$ & 2.2 & 27.3 & 32.0 & 0.65 & 0.05 & 0.45 & 0.75 & 0.25 & 1.5 & 0.65 & 31.4 \\
\hline $\mathrm{H}_{2} \mathrm{O}$ & 12.8 & 4.8 & 3.8 & 10.0 & 11.4 & 7.0 & 7.2 & 5.8 & 6.4 & 10.4 & 3.0 \\
\hline $\mathrm{Sr}$ & 0.013 & 0.11 & 0.13 & 0.12 & 0.026 & 0.005 & 0.006 & 0.005 & 0.013 & 0.009 & 0.1 \\
\hline $\mathrm{Ba}$ & 0.13 & 0.05 & 0.045 & 0.028 & 0.075 & 0.07 & 0.11 & 0.1 & 0.27 & 0.32 & 0.004 \\
\hline $\mathrm{Li}$ & 0.005 & 0.002 & 0.002 & 0.004 & 0.004 & 0.001 & 0.003 & 0.003 & 0.003 & 0.005 & 0.001 \\
\hline $\mathrm{Cu}$ & 0.07 & 0.0025 & 0.003 & 0.015 & 0.022 & 0.0055 & 0.009 & 0.0115 & 0.006 & 0.0085 & 0.021 \\
\hline $\mathrm{Pb}$ & 0.0011 & 0.0001 & 0.0015 & 0.0015 & 0.004 & 0.0003 & 0.0025 & 0.0015 & 0.011 & 0.002 & 0.001 \\
\hline $\mathrm{Zn}$ & 0.014 & 0.0055 & 0.0065 & 0.018 & 0.015 & 0.0055 & 0.0105 & 0.005 & 0.009 & 0.013 & 0.004 \\
\hline Co & 0.0035 & 0.0004 & 0.0001 & 0.0085 & 0.012 & 0.0018 & 0.002 & 0.0008 & 0.0018 & 0.005 & 0.0001 \\
\hline
\end{tabular}

Note: Each sample has the following properties:

(21) depth, 17.9m, Unit 1, Quaternary, Radiolarian clay; (22) depth, 52.8m, Unit 2, upper Miocene or younger, nanno ooze; (23) depth, 102.4m, Unit 2, upper Miocene or younger, nanno ooze; (24) depth, 172.8m, Unit 3A, Upper Cretaceous, clay; (25) depth, 197.8m, Unit 3A, Upper Cretaceous, zeolitebearing clay; (26) depth, 255.6m, Unit 3B, Cretaceous, claystone; (27) depth, 350.3m, Unit 3B, Cretaceous, claystone; (28) depth, 407.3m, Unit 3B, lower Cretaceous, claystone; (29) depth, 472.3m, Unit 4, Lower Cretaceous, claystone and clay; (30) depth, 510.3m, Unit 4, Upper Jurassic-Lower Cretaceous, claystone; (31) depth, 528.1m, Unit 4, Upper Jurassic-Lower Cretaceous, nanno claystone. 
TABLE 4

Major and Trace Element Analyses, Site 262

\begin{tabular}{|c|c|c|c|c|c|c|c|c|c|c|c|c|c|c|c|}
\hline \multirow[b]{2}{*}{ Element } & \multicolumn{15}{|c|}{ Sample } \\
\hline & 32 & 33 & 34 & 35 & 36 & 37 & 38 & 39 & 40 & 41 & 42 & 43 & 44 & 45 & 46 \\
\hline \multicolumn{16}{|l|}{ Major } \\
\hline $\mathrm{SiO}_{2}$ & 28.4 & 37.0 & 40.0 & 38.5 & 31.6 & 27.8 & 19.3 & 14.4 & 18.3 & 11.0 & 9.3 & 1.5 & 1.7 & 1.5 & 1.3 \\
\hline $\mathrm{A}_{2}{ }_{2} \mathrm{O}_{3}$ & 8.7 & 8.7 & 9.5 & 10.4 & 9.6 & 8.6 & 6.7 & 3.6 & 3.9 & 2.3 & 2.5 & 0.1 & 0.1 & 0.1 & 0.1 \\
\hline $\mathrm{Fe}_{2} \mathrm{O}_{3}$ & 3.3 & 3.9 & 4.0 & 4.1 & 3.7 & 3.4 & 2.3 & 2.1 & 2.0 & 1.1 & 1.15 & 0.4 & 0.3 & 0.2 & 0.5 \\
\hline $\mathrm{CaO}$ & 24.5 & 19.6 & 17.9 & 17.5 & 22.2 & 27.0 & 32.0 & 38.0 & 37.0 & 42.0 & 43.0 & 43.5 & 44.0 & 44.0 & 44.0 \\
\hline $\mathrm{MgO}$ & 2.4 & 1.9 & 2.0 & 2.2 & 1.7 & 1.7 & 1.0 & 3.0 & 2.6 & 2.5 & 2.6 & 9.0 & 4.9 & 8.0 & 8.0 \\
\hline $\mathrm{Na}_{2} \mathrm{O}$ & 2.1 & 1.8 & 2.2 & 2.3 & 2.0 & 1.55 & 1.5 & 1.5 & 1.6 & 0.95 & 1.05 & 0.78 & 0.82 & 0.56 & 0.74 \\
\hline $\mathrm{K}_{2} \mathrm{O}$ & 1.3 & 1.4 & 1.6 & 1.7 & 1.6 & 1.4 & 1.1 & 0.8 & 0.95 & 0.5 & 0.5 & 0.1 & 0.13 & 0.16 & 0.1 \\
\hline $\mathrm{MnO}$ & 0.05 & 0.11 & 0.12 & 0.14 & 0.09 & 0.08 & $<0.02$ & $<0.02$ & $<0.02$ & $<0.02$ & $<0.02$ & $<0.02$ & $<0.02$ & $<0.02$ & $<0.02$ \\
\hline $\mathrm{P}_{2} \mathrm{O}_{5}$ & 0.06 & 0.17 & 0.1 & 0.11 & 0.1 & 0.14 & 0.13 & 0.17 & 0.14 & 0.18 & 0.21 & 0.1 & 0.14 & 0.02 & 0.12 \\
\hline $\mathrm{TiO}_{2}$ & 0.35 & 0.35 & 0.39 & 0.4 & 0.36 & 0.31 & 0.28 & 0.16 & 0.18 & 0.1 & 0.1 & 0.01 & 0.02 & 0.02 & 0.01 \\
\hline $\mathrm{Cr}_{2} \mathrm{O}_{3}$ & $<0.1$ & $<0.15$ & $<0.1$ & $<0.1$ & $<0.1$ & $<0.1$ & $<0.1$ & $<0.1$ & $<0.1$ & $<0.1$ & $<0.1$ & $<0.1$ & $<0.1$ & 0.15 & $<0.1$ \\
\hline $\mathrm{v}_{2} \mathrm{O}_{5}$ & $<0.05$ & $<0.05$ & $<0.05$ & $<0.05$ & $<0.05$ & $<0.05$ & $<0.05$ & $<0.05$ & $<0.05$ & $<0.05$ & $<0.05$ & $<0.05$ & $<0.05$ & $<0.05$ & $<0.05$ \\
\hline LOI & 29.5 & 24.7 & 22.3 & 22.5 & 27.1 & 28.1 & 35.9 & 36.3 & 34.0 & 38.1 & 39.0 & 42.3 & 44.6 & 44.8 & 44.4 \\
\hline Total & 100.7 & 99.8 & 100.1 & 99.9 & 100.1 & 100.1 & 100.2 & 100.0 & 100.7 & 98.7 & 99.4 & 97.7 & 96.7 & 99.5 & 99.2 \\
\hline \multicolumn{16}{|l|}{ Trace } \\
\hline $\mathrm{CO}_{2}$ & 20.5 & 15.3 & 13.3 & 13.6 & 17.2 & 21.3 & 25.1 & 31.3 & 29.3 & 35.4 & 35.0 & 38.8 & 38.7 & 42.9 & 41.4 \\
\hline $\mathrm{H}_{2} \mathrm{O}$ & 6.8 & 6.4 & 6.5 & 7.2 & 9.2 & 4.2 & 5.7 & 4.3 & 4.4 & 3.0 & 2.0 & 1.0 & 1.0 & 1.2 & 1.2 \\
\hline $\mathrm{Sr}$ & 0.1 & 0.085 & 0.075 & 0.06 & 0.075 & 0.105 & 0.14 & 0.27 & 0.21 & 0.18 & 0.17 & 0.055 & 0.06 & 0.06 & 0.006 \\
\hline $\mathrm{Ba}$ & 0.46 & 0.045 & 0.044 & 0.045 & 0.043 & 0.045 & 0.041 & 0.014 & 0.010 & 0.005 & 0.004 & 0.002 & 0.002 & 0.002 & 0.001 \\
\hline $\mathrm{Li}$ & 0.003 & 0.003 & 0.003 & 0.003 & 0.003 & 0.003 & 0.002 & 0.002 & 0.002 & 0.001 & 0.001 & $<0.001$ & $<0.001$ & 0.001 & $<0.001$ \\
\hline $\mathrm{Cu}$ & 0.004 & 0.004 & 0.004 & 0.004 & 0.004 & 0.004 & 0.003 & 0.0018 & 0.0015 & 0,001 & 0.0008 & 0.0003 & 0.0002 & 0.0003 & 0.0005 \\
\hline $\mathrm{Pb}$ & 0.001 & 0.0013 & 0.0001 & 0.0015 & 0.007 & 0.007 & 0.0001 & 0,0015 & 0.0015 & 0.0008 & 0.0001 & $<0.0001$ & $<0.0001$ & 0.0002 & 0.0005 \\
\hline $\mathrm{Z}_{\mathrm{r}}$ & 0.0075 & 0.009 & 0.0095 & 0.0095 & 0.009 & 0.009 & 0.0065 & 0.0055 & 0.0045 & 0.003 & 0.003 & 0.0008 & 0.0008 & 0.0013 & 0.0018 \\
\hline Co & $<0.0001$ & 0.0008 & 0.0004 & 0.0008 & 0.0008 & 0.0008 & 0.0004 & 0.0008 & $<0.0001$ & $<0.0001$ & 0.0002 & 0.0001 & $<0.0001$ & 0.0001 & 0.0001 \\
\hline $\mathrm{Ni}$ & 0.003 & 0.0035 & 0.0035 & 0.004 & 0.004 & 0.0035 & 0.0025 & 0.002 & 0.0015 & 0.001 & 0.0003 & 0.0001 & 0.0001 & 0.0015 & 0.0005 \\
\hline
\end{tabular}

Note: Each sample has the following properties:

(32) depth, 3.8m, Unit 1, Pleistocene-Holocene, foram-rich nanno ooze; (33) depth, 52.5m, Unit 1, Pleistocene-Holocene, rad-rich nanno ooze; (34) depth, 72.1m, Unit, PleistoceneHolocene, rad-rich nanno ooze; (35) depth, $127.0 \mathrm{~m}$, Unit 1, Pleistocene-Holocene, clay rad-rich nanno ooze; (36) depth, 207.8m, Unit 1, Pleistocene-Holocene, clay-nanno ooze; (37) depth, 247.0m, Unit 1, Quaternary clay-rich nanno ooze; (38) depth, 255.3m, Unit 1, Quaternary, foram and clay-rich nanno ooze; (39) depth, 323.3m, Unit 2, Pleistocene clay nanno ooze; (40) depth, 347.3m, Unit 3, Pliocene, nanno-rich foram ooze; (41) depth, 362.5m, Unit 3, Pliocene, nanno foram ooze; (42) depth, 395.0m, Unit 3, Pliocene, micarb foram ooze; (43) depth, $416.8 \mathrm{~m}$, Unit 4, Pliocene, foram-rich micarb ooze; (44) depth, $420.0 \mathrm{~m}$, Unit 4, Pliocene, foram-rich dolomite; (45) dpeth, 421.0m, Unit 4, Pliocene, foramrich dolomite; (46) depth, 421.2m, Unit 4, Pliocene, foram-rich dolomite. 
TABLE 5

Major and Trace Element Analyses, Site 263

\begin{tabular}{|c|c|c|c|c|c|c|c|c|c|c|}
\hline \multirow[b]{2}{*}{ Element } & \multicolumn{10}{|c|}{ Sample } \\
\hline & 47 & 48 & 49 & 50 & 51 & 52 & 53 & 54 & 55 & 56 \\
\hline \multicolumn{11}{|l|}{ Major } \\
\hline $\mathrm{SiO}_{2}$ & 5.8 & 15.2 & 56.5 & 56.0 & 62.5 & 65.0 & 59.0 & 47.2 & 60.0 & 57.0 \\
\hline $\mathrm{Al}_{2} \mathrm{O}_{3}$ & 0.6 & 3.2 & 12.7 & 13.1 & 13.5 & 12.5 & 16.7 & 14.6 & 12.4 & 13.7 \\
\hline $\mathrm{Fe}_{2} \mathrm{O}_{3}$ & 0.9 & 1.8 & 5.4 & 6.2 & 6.4 & 6.4 & 6.9 & 14.3 & 7.2 & 9.3 \\
\hline $\mathrm{CaO}$ & 48.0 & 39.5 & 3.6 & 3.8 & 1.2 & 1.2 & 1.3 & 3.6 & 4.2 & 2.2 \\
\hline $\mathrm{MgO}$ & 0.95 & 2.0 & 2.4 & 2.4 & 2.0 & 1.8 & 1.9 & 2.1 & 1.15 & 2.1 \\
\hline $\mathrm{Na}_{2} \mathrm{O}$ & 1.5 & 1.45 & 2.35 & 2.2 & 2.05 & 1.65 & 1.4 & 1.0 & 0.76 & 1.1 \\
\hline $\mathrm{K}_{2} \mathrm{O}$ & 0.2 & 0.65 & 2.4 & 2.4 & 1.9 & 1.9 & 2.7 & 1.9 & 1.7 & 2.05 \\
\hline $\mathrm{MnO}$ & $<0.03$ & $<0.03$ & 0.03 & 0.07 & 0.17 & 0.02 & 0.03 & 0.11 & 0.1 & 0.05 \\
\hline $\mathrm{P}_{2} \mathrm{O}_{5}$ & 0.08 & 0.14 & 0.11 & 0.17 & 0.12 & 0.09 & 0.23 & 0.54 & 0.11 & 0.2 \\
\hline $\mathrm{TiO}_{2}$ & 0.05 & 0.17 & 0.6 & 0.59 & 0.57 & 0.56 & 0.88 & 0.81 & 0.4 & 0.78 \\
\hline $\mathrm{Cr}_{2} \mathrm{O}_{3}$ & $<0.1$ & $<0.1$ & $<0.1$ & $<0.1$ & $<0.1$ & $<0.1$ & $<0.1$ & $<0.1$ & $<0.1$ & $<0.1$ \\
\hline $\mathrm{V}_{2} \mathrm{O}_{5}$ & $<0.05$ & $<0.05$ & $<0.05$ & $<0.05$ & $<0.05$ & $<0.05$ & $<0.05$ & $<0.05$ & $<0.05$ & $<0.05$ \\
\hline LOI & 40.9 & 36.5 & 13.7 & 13.4 & 9.6 & 9.2 & 8.8 & 14.2 & 12.0 & 11.3 \\
\hline Total & 99.0 & 100.6 & 99.8 & 100.3 & 100.0 & 100.3 & 99.8 & 100.4 & 100.0 & 99.8 \\
\hline \multicolumn{11}{|l|}{ Trace } \\
\hline $\mathrm{CO}_{2}$ & 37.9 & 31.4 & 2.7 & 2.9 & 2.0 & 0.65 & 0.25 & 6.1 & 2.9 & 1.2 \\
\hline $\mathrm{H}_{2} \mathrm{O}$ & 3.0 & 4.3 & 11.4 & 10.5 & 8.4 & 6.8 & 7.2 & 7.0 & 7.7 & 6.6 \\
\hline $\mathrm{Sr}$ & 0.12 & 0.21 & 0.012 & 0.01 & 0.007 & 0.008 & 0.007 & 0.008 & 0.005 & 0.008 \\
\hline $\mathrm{Ba}$ & 0.013 & 0.02 & 0.02 & 0.023 & 0.033 & 0.041 & 0.036 & 0.035 & 0.02 & 0.03 \\
\hline $\mathrm{Li}$ & $<0.001$ & 0.002 & 0.004 & 0.003 & 0.005 & 0.006 & 0.011 & 0.010 & 0.003 & 0.005 \\
\hline $\mathrm{Cu}$ & 0.0012 & 0.0025 & 0.0055 & 0.0055 & 0.0055 & 0.0065 & 0.0035 & 0.004 & 0.0015 & 0.004 \\
\hline $\mathrm{Pb}$ & $<0.0001$ & 0.0005 & 0.0015 & 0.002 & 0.0018 & 0.0018 & 0.002 & 0.002 & 0.002 & 0.0018 \\
\hline $\mathrm{Zn}$ & 0.005 & 0.004 & 0.013 & 0.014 & 01016 & 0.023 & 0.0125 & 0.026 & 0.018 & 0.02 \\
\hline Co & 0.0003 & 0.0005 & 0.0025 & 0.0025 & 0.002 & 0.0015 & 0.002 & 0.001 & 0.0003 & 0.0008 \\
\hline $\mathrm{Ni}$ & 0.0008 & 0.002 & 0.0055 & 0.0013 & 0.001 & 0.001 & 0.001 & 0.001 & 0.001 & 0.0045 \\
\hline
\end{tabular}

Note: Each sample has the following properties:

(47) depth, 2.3m, Unit 1, Quaternary, detrital foram nanno ooze; (48) depth, 57.7m, Unit 1, Quaternary, detrital foram nanno ooze; (49) depth, $114.3 \mathrm{~m}$, Unit 2, Cretaceous, nanno-bearing clay; (50) depth, $152.3 \mathrm{~m}$, Unit 2, Cretaceous, nanno-bearing clay; (51) depth, 263.3m, Unit 3, Cretaceous, clay; (52) depth, 392.5m, Unit 3, Lower Cretaceous, clay; (53) depth, 483.9m, Unit 4, Lower Cretaceous, clay; (54) depth, 635.4m, Unit 4, Lower Cretaceous, clay; (55) depth, 736.0m, Unit 4, Lower Cretaceous, clay; (56) depth, 741.2m, Unit 4, Lower Cretaceous, clay.

by Olson and Miller (1958) and Chayes (1960). In such a system most components will inevitably show a negative correlation with the dominant component, in this case $\mathrm{CaCO}_{3}$.

Where correlation matrices are obtained for the various groupings, again the Cenozoic and calcareous ooze matrices (Tables 11 and 13) tend to be dominated by $\mathrm{CaO}, \mathrm{CO}_{2}$, and associated variables, making it difficult if not impossible to recognize any meaningful correlations. The carbonate-poor Mesozoic and clay groups, on the other hand, are not dominanted by calcium carbonate and despite the closed array effect, some significant correlations are evident (Tables 10 and 12). Perhaps the most important is the negative correlation of $\mathrm{SiO}_{2}$ with $\mathrm{TiO}_{2}, \mathrm{Fe}_{2} \mathrm{O}_{3}, \mathrm{~K}_{2} \mathrm{O}, \mathrm{H}_{2} \mathrm{O}$, and $\mathrm{Zn}$. This is probably the result of sedimentary dilution of terrigenous and some chemical sediments by the influx of biogenic silica.
The basic groupings of clay (predominantly Mesozoic) and calcareous ooze (predominantly Cenozoic) are found to have opposed correlation coefficients in a number of cases. Some example of this are shown in Figure 4, with the two groups having markedly different regression lines. Opposed correlation coefficients are also apparent in Figure 5, where significant correlations (assuming $\rho \geq \pm 0.80$ and $\alpha=0.05$ ) for clay, calcareous ooze, Mesozoic, and Cenozoic groups of sediments are shown schematically. There are, in addition, a number of correlations which are significant and positive in at least three of these four groups. Many are to be expected such as $\mathrm{CaO}-\mathrm{CO}_{2}$ and $\mathrm{Co}-\mathrm{Ni}$; others are not quite so obvious such as $\mathrm{Cu}-\mathrm{MnO}$ and $\mathrm{Zn}-\mathrm{Al}_{2} \mathrm{O}_{3}$; some are perhaps unexpected such as $\mathrm{P}_{2} \mathrm{O}_{5}-\mathrm{Fe}_{2} \mathrm{O}_{3}$, but this could be due to trace amounts of vivianite. The lack of significant correlations is also notable in some cases, e.g., the absence of $\mathrm{Sr}-\mathrm{CaO}$ and $\mathrm{P}_{2} \mathrm{O}_{5}-\mathrm{CaO}$ correlations. 
TABLE 6

Average Major Element Composition of Leg 27 and Other Deep-Sea Sediments

\begin{tabular}{|c|c|c|c|c|c|c|}
\hline & \multicolumn{3}{|c|}{ Average Leg 27 Compositions } & \multicolumn{3}{|c|}{ Average Pelagic Sediments ${ }^{a}$} \\
\hline & $\begin{array}{l}\text { Mesozoic } \\
\text { Sediments } \\
(\%)\end{array}$ & $\begin{array}{l}\text { Cenozoic } \\
\text { Sediments } \\
(\%)\end{array}$ & $\begin{array}{c}\text { Timor } \\
\text { Trough } \\
\text { Sediments } \\
(\%)\end{array}$ & $\begin{array}{l}\text { Calcareous } \\
\text { Sediments } \\
(\%)\end{array}$ & $\begin{array}{l}\text { Argillaceous } \\
\text { Sediments } \\
(\%)\end{array}$ & $\begin{array}{l}\text { Siliceous } \\
\text { Sediments } \\
\text { (\%) }\end{array}$ \\
\hline $\mathrm{SiO}_{2}$ & 58.6 & 23.0 & 18.8 & 27.0 & 55.4 & 64.0 \\
\hline $\mathrm{A}_{2}{ }_{2} \mathrm{O}_{3}$ & 10.4 & 6.1 & 5.0 & 8.0 & 17.6 & 13.4 \\
\hline $\mathrm{Fe}_{2} \mathrm{O}_{3}$ & 6.2 & 2.7 & 2.2 & 3.9 & 8.3 & 6.3 \\
\hline $\mathrm{CaO}$ & 4.4 & 30.8 & 33.1 & 28.5 & 1.4 & 1.6 \\
\hline $\mathrm{MgO}$ & 2.1 & 2.9 & 3.6 & 2.3 & 3.8 & 2.5 \\
\hline $\mathrm{Na}_{2} \mathrm{O}$ & 2.12 & 1.86 & 1.43 & 0.8 & 1.52 & 0.94 \\
\hline $\mathrm{K}_{2} \mathrm{O}$ & 2.16 & 1.10 & 0.87 & 1.48 & 3.26 & 1.90 \\
\hline $\mathrm{MnO}$ & 0.29 & 0.17 & 0.05 & 0.32 & 0.47 & 0.41 \\
\hline $\mathrm{P}_{2} \mathrm{O}_{5}$ & 0.14 & 0.14 & 0.13 & 0.15 & 0.14 & 0.27 \\
\hline $\mathrm{TiO}_{2}$ & 0.52 & 0.25 & 0.20 & 0.44 & 0.84 & 0.65 \\
\hline $\mathrm{Cr}_{2} \mathrm{O}_{3}$ & $<0.1$ & $<0.1$ & $<0.1$ & - & - & - \\
\hline $\mathrm{V}_{2} \mathrm{O}_{5}$ & $<0.05$ & $<0.05$ & $<0.05$ & - & - & - \\
\hline $\mathrm{CO}_{2}$ & 4.75 & 25.4 & 27.8 & 23.3 & 0.77 & 0.93 \\
\hline $\mathrm{H}_{2} \mathrm{O}$ & 8.32 & 5.60 & 4.3 & 3.9 & 6.5 & 7.1 \\
\hline
\end{tabular}

On the basis of the correlation matrices (Tables 9-13, Figure 5) and the X-ray diffraction data, it is possible to group the oxides and elements into a biogenic carbonate association $\left(\mathrm{CaO}, \mathrm{CO}_{2}, \mathrm{Sr}[?]\right)$; biogenic silica association $\left(\mathrm{SiO}_{2}\right)$; clay and terrigenous association $\left(\mathrm{SiO}_{2}, \mathrm{Al}_{2} \mathrm{O}_{3}\right.$, $\mathrm{TiO}_{2}, \mathrm{Fe}_{2} \mathrm{O}_{3}, \mathrm{Na}_{2} \mathrm{O}, \mathrm{H}_{2} \mathrm{O}, \mathrm{Zn}$ ); and ferromanganese

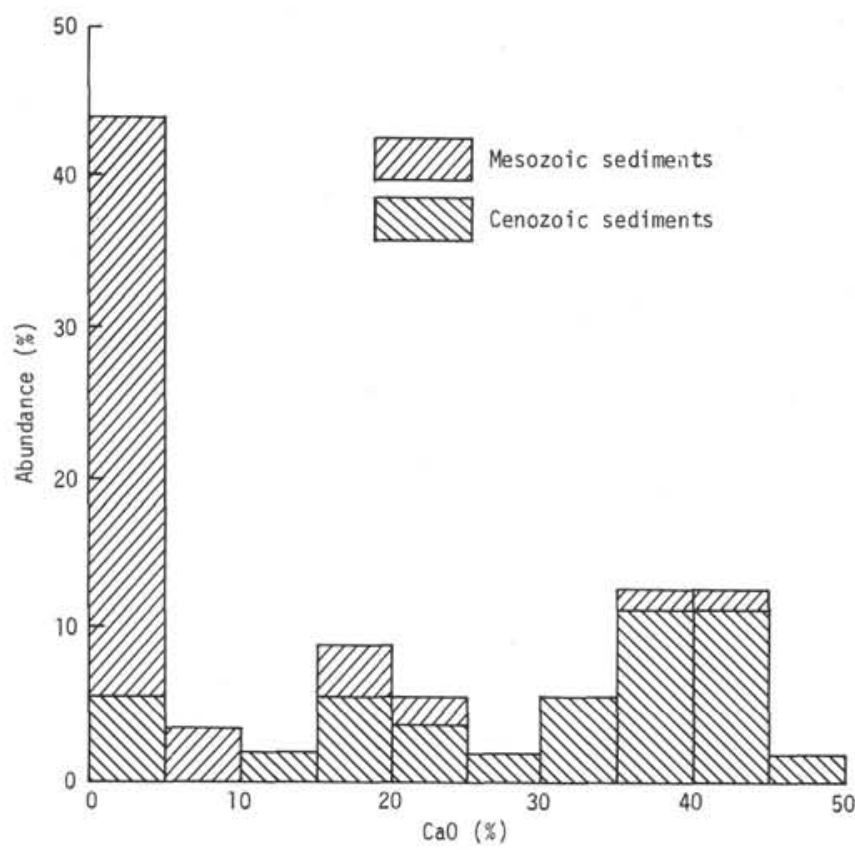

Figure 3. Histogram indicating the percentage of $\mathrm{CaO}$ in Mesozoic and Cenozoic sediments, Leg 27, eastern Indian Ocean. association $\left(\mathrm{MnO}, \mathrm{Fe}_{2} \mathrm{O}_{3}, \mathrm{Cu}, \mathrm{Co}, \mathrm{Ni}\right)$. For a number of elements there is no clear association.

In an attempt to further identify interelement associations and groupings, R-mode factor analysis was undertaken on the two basic types of Leg 27 sediments-clay (containing less than $10 \% \mathrm{CaO}$ ) and calcareous ooze (containing more than $10 \% \mathrm{CaO}$ ). This technique has previously been applied to sedimentary geochemical data by, for instance, Hirst and Kaye (1971) and Summerhayes (1972) in order to obtain meaningful groupings of large numbers of variables.

The multivariate analysis program BMD03M of Dixon (1971), which incorporates the Varimax factor rotation, was used. An arbitrary cutoff of six factors was specified. In addition, factor loadings of less than \pm 0.4 were considered to be not significant. The resultant factor loadings are shown diagrammatically in Figures 6 and 7.

Considering the factor loadings for calcareous ooze, the six factors in Figure 6 are found to explain $91.8 \%$ of the total variance of the original data. As mentioned previously, however, interelement associations are effectively masked by the abundance of calcium carbonate in the sediment. This is apparent in Factor 1 of Figure 6 in which only $\mathrm{CaO}$ and $\mathrm{CO}_{2}$ have a positive factor loading, and all other variables have a negative factor loading. Thus, Factor 1 which accounts for $59 \%$ of the variance is merely a function of the closed array effect previously discussed and consequently is not of any real significance.

Factor 2 corresponds fairly well to the ferromanganese-associated variables previously recognized from qualitative consideration of the correlations indicated by the correlation matrices. The 
TABLE 7

Average Composition of Sediments on a Carbonate-Free, Water-Free Basis

\begin{tabular}{l|ccc|ccc}
\cline { 2 - 7 } & \multicolumn{2}{c|}{ Average Leg 27 Compositions } & \multicolumn{3}{c}{ Average Pelagic Sediments } \\
\cline { 2 - 7 } & $\begin{array}{c}\text { Mesozoic } \\
\text { Sediments } \\
(\%)\end{array}$ & $\begin{array}{c}\text { Cenozoic } \\
\text { Sediments } \\
(\%)\end{array}$ & $\begin{array}{c}\text { Timor } \\
\text { Trough } \\
\text { Sediments } \\
(\%)\end{array}$ & $\begin{array}{c}\text { Calcareous } \\
\text { Sediments } \\
(\%)\end{array}$ & $\begin{array}{c}\text { Argillaceous } \\
\text { Sediments } \\
(\%)\end{array}$ & $\begin{array}{c}\text { Siliceous } \\
\text { Sediments } \\
(\%)\end{array}$ \\
\hline $\mathrm{SiO}_{2}$ & 72.2 & 62.3 & 60.3 & 60.5 & 61.0 & 71.1 \\
$\mathrm{A1}_{2} \mathrm{O}_{3}$ & 12.7 & 16.4 & 16.0 & 18.5 & 19.3 & 14.9 \\
$\mathrm{Fe}_{2} \mathrm{O}_{3}$ & 7.6 & 7.5 & 7.1 & 9.9 & 9.0 & 7.2 \\
$\mathrm{MgO}$ & 1.1 & 3.5 & 0.0 & 3.1 & 3.8 & 2.2 \\
$\mathrm{Na}_{2} \mathrm{O}$ & 2.60 & 5.32 & 4.58 & 2.52 & 1.69 & 0.94 \\
$\mathrm{~K}_{2} \mathrm{O}$ & 2.66 & 3.14 & 2.79 & 3.32 & 3.60 & 2.11 \\
$\mathrm{MnO}^{n}$ & 0.35 & 0.75 & 0.16 & 0.76 & 0.53 & 0.56 \\
$\mathrm{P}_{2} \mathrm{O}_{5}$ & 0.17 & 0.36 & 0.42 & 0.46 & 0.16 & 0.30 \\
$\mathrm{TiO}_{2}$ & 0.64 & 0.68 & 0.64 & 0.95 & 0.93 & 0.73 \\
\hline
\end{tabular}

${ }^{\mathrm{a}}$ Average pelagic values recalculated from El Wakeel and Riley (1961)

TABLE 8

Average Trace Element Composition of Leg 27 and Other Deep-Sea Sediments

\begin{tabular}{l|cc|ccc}
\cline { 2 - 6 } & \multicolumn{2}{|c|}{ Average Leg 27 Compositions } & \multicolumn{3}{|c}{ Average Pelagic Sediments } \\
\cline { 2 - 6 } & $\begin{array}{c}\text { Mesozoic } \\
\text { Sediments } \\
\text { (ppm) }\end{array}$ & $\begin{array}{c}\text { Cenozoic } \\
\text { Sediments } \\
\text { (ppm) }\end{array}$ & $\begin{array}{c}\text { Pacific }^{\mathrm{a}} \\
\text { Sediments }^{(\mathrm{ppm})}\end{array}$ & $\begin{array}{c}\text { Carbonate }^{\mathrm{b}} \\
(\mathrm{ppm})\end{array}$ & $\begin{array}{c}\text { Clay }^{\mathrm{b}} \\
(\mathrm{ppm})\end{array}$ \\
\hline $\mathrm{Sr}$ & 204 & 990 & 710 & 2000 & 180 \\
$\mathrm{Ba}$ & 768 & 500 & 390 & 190 & 2300 \\
$\mathrm{Li}$ & 38 & 30 & 59 & - & 2.6 \\
$\mathrm{Cu}$ & 133 & 21 & 740 & 30 & 250 \\
$\mathrm{~Pb}$ & 21 & 12 & 150 & 9 & 80 \\
$\mathrm{Zn}$ & 127 & 85 & - & 35 & 165 \\
$\mathrm{Co}$ & 23 & 18 & 160 & 7 & 74 \\
$\mathrm{Ni}$ & 54 & 75 & 320 & 30 & 225 \\
\hline
\end{tabular}

${ }^{a}$ Average values for Pacific pelagic sediments after Goldberg and Arrhenius (1958)

${ }^{b}$ Average values for carbonates and clay from Turekian and Wedepohl (1961)

oxides and elements, $\mathrm{SiO}_{2}, \mathrm{Fe}_{2} \mathrm{O}_{3}, \mathrm{MnO}, \mathrm{Ba}, \mathrm{Cu}, \mathrm{Zn}$, $\mathrm{Co}, \mathrm{Ni}$, are included in this factor. This association is probably attributable to oxide coatings of iron and manganese, or in some instances, to nodules of iron and manganese oxide which are able to adsorb large amounts of other elements from seawater (Krauskopf, 1956). This factor is responsible for approximately $11 \%$ of the total variance.

Factor 3, which accounts for $7 \%$ of the total variance, is rather puzzling as it groups together the two other dissimilar variables $\mathrm{P}_{2} \mathrm{O}_{5}$ and $\mathrm{Sr}$. It is possible that this is a function of biogenic activity, but there is no sympathetic association of $\mathrm{CaO}$ and $\mathrm{CO}_{2}$ and the factor loadings for $\mathrm{CaO}$ and $\mathrm{CO}_{2}$ are -0.11 and -0.12 , respectively.

Similarly, Factor 5 is a function primarily of $\mathrm{MgO}$ and $\mathrm{Sr}$ with, again, the most likely controlling factor being with the biota. Strontium is more abundant in high-magnesian calcite than in low-magnesian calcite, but as some of the calcareous sediments being dealt with here are from below the lysocline, the normal chemical conditions may not apply. Consequently, although both Factors 3 and 5 are perhaps in part a response to biogenic activity, the reasons for such an association are not clear.

Factor 6 has the unlikely variable combination of $\mathrm{Pb}$ and $\mathrm{H}_{2} \mathrm{O}$; it is probable that most of the water is present in the clays, consequently, it is possible that Factor 6 is a reflection of the adsorption of lead by clays. Factor 4 (res) is primarily a function of residual volatile material, excluding $\mathrm{H}_{2} \mathrm{O}$ and $\mathrm{CO}_{2}$ (res); it is not considered to be geologically significant.

Factor scores are a method of showing sample to sample relations which result from the effects of the factors previously obtained by R-mode factor analysis. Dixon (1971) discusses the mathematical basis of factor scores. This intuitive method has been applied to geochemical data (Summerhayes, 1972), particularly to areal variations, but here it is applied to vertical sequences. 

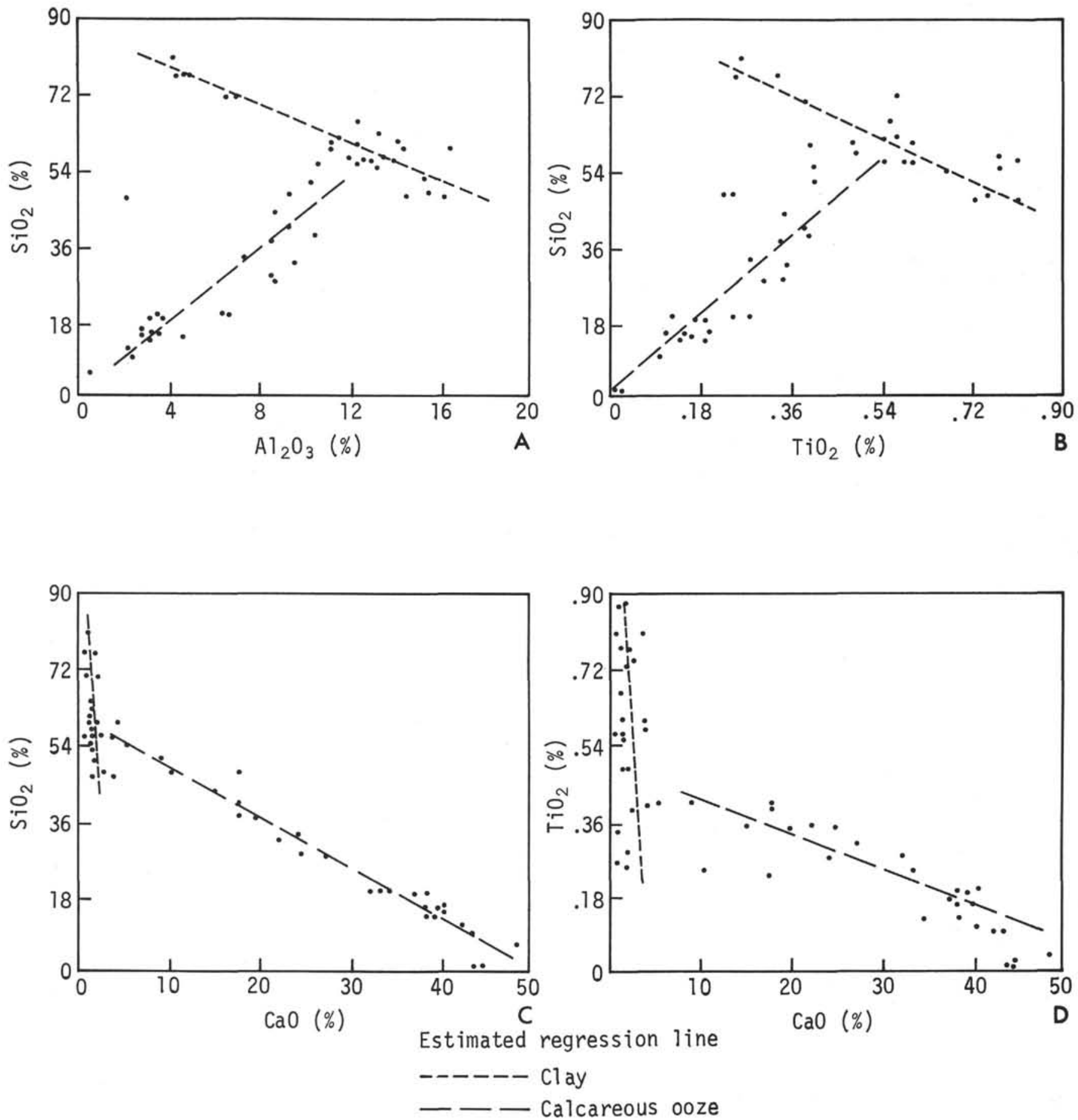

Figure 4. X-Y plots illustrating the different distribution patterns of clays (predominantly Mesozoic) and calcareous ooze (predominantly (Cenozoic), (A) $\mathrm{SiO}_{2}$ plotted against $\mathrm{Al}_{2} \mathrm{O}_{3} ;$ (B) $\mathrm{SiO}_{2}$ plotted against $\mathrm{TiO}_{2} ;(\mathrm{C}) \mathrm{SiO}_{2}$ plotted against $\mathrm{CaO} ;(\mathrm{D})$ $\mathrm{TiO}_{2}$ plotted against $\mathrm{CaO}$. 
TABLE 9

\begin{tabular}{|c|c|c|c|c|c|c|c|c|c|c|c|c|c|c|c|c|c|c|c|c|c|}
\hline & $\mathrm{SiO}_{2}$ & $\mathrm{TiO}_{2}$ & $\mathrm{Al}_{2} \mathrm{O}_{3}$ & $\mathrm{Fe}_{2} \mathrm{O}_{3}$ & MnO & $\mathrm{MgO}$ & $\mathrm{CaO}$ & $\mathrm{Na}_{2} \mathrm{O}$ & $\mathrm{K}_{2} \mathrm{O}$ & $\mathrm{P}_{2} \mathrm{O}_{5}$ & $\mathrm{H}_{2} \mathrm{O}$ & Res & $\mathrm{CO}_{2}$ & St & $\mathrm{Ba}$ & $\mathrm{Li}$ & $\mathrm{Cu}$ & $\mathrm{Pb}$ & $\mathrm{Zn}$ & Co & $\mathrm{Ni}$ \\
\hline $\mathrm{SiO}_{2}$ & 1.000 & & & & & & & & & & & & & & & & & & & & \\
\hline $\mathrm{TiO}_{2}$ & 0.682 & 1.000 & & & & & & & & & & & & & & & & & & & \\
\hline $\mathrm{Al}_{2} \mathrm{O}_{3}$ & 0.641 & 0.924 & 1.000 & & & & & & & & & & & & & & & & & & \\
\hline $\mathrm{Fe}_{2} \mathrm{O}_{3}$ & 0.698 & 0.890 & 0.817 & 1.000 & & & & & & & & & & & & & & & & & \\
\hline $\mathrm{MnO}$ & 0.256 & 0.396 & 0.393 & 0.352 & 1.000 & & & & & & & & & & & & & & & & \\
\hline $\mathrm{MgO}$ & -0.314 & -0.157 & -0.209 & -0.198 & 0.019 & 1.000 & & & & & & & & & & & & & & & \\
\hline $\mathrm{CaO}$ & -0.960 & -0.827 & -0.808 & -0.815 & -0.359 & 0.215 & 1.000 & & & & & & & & & & & & & & \\
\hline $\mathrm{Na}_{2} \mathrm{O}$ & 0.462 & 0.465 & 0.557 & 0.327 & 0.518 & -0.234 & -0.552 & 1.000 & & & & & & & & & & & & & \\
\hline $\mathrm{K}_{2} \mathrm{O}$ & 0.757 & 0.853 & 0.838 & 0.793 & 0.450 & -0.210 & -0.866 & 0.688 & 1.000 & & & & & & & & & & & & \\
\hline $\mathrm{P}_{2} \mathrm{O}_{5}$ & 0.112 & 0.481 & 0.442 & 0.527 & 0.368 & 0.128 & -0.263 & 0.053 & 0.324 & 1.000 & & & & & & & & & & & \\
\hline $\mathrm{H}_{2} \mathrm{O}$ & 0.683 & 0.789 & 0.862 & 0.689 & 0.556 & -0.196 & -0.817 & 0.785 & 0.875 & 0.343 & 1.000 & & & & & & & & & & \\
\hline Res & -0.451 & -0.270 & -0.204 & -0.270 & -0.273 & 0.008 & 0.398 & -0.177 & -0.316 & -0.071 & -0.357 & 1.000 & & & & & & & & & \\
\hline $\mathrm{CO}_{2}$ & -0.961 & -0.825 & -0.812 & -0.809 & -0.366 & 0.289 & 0.995 & -0.573 & -0.879 & -0.256 & -0.830 & 0.386 & 1.000 & & & & & & & & \\
\hline St & -0.702 & -0.570 & -0.531 & -0.604 & -0.351 & -0.012 & 0.738 & -0.319 & -0.595 & -0.085 & -0.529 & 0.214 & 0.713 & 1.000 & & & & & & & \\
\hline $\mathrm{Ba}$ & 0.573 & 0.146 & 0.156 & 0.216 & 0.328 & -0.164 & -0.487 & 0.312 & 0.324 & 0.070 & 0.321 & -0.327 & -0.493 & -0.373 & 1.000 & & & & & & \\
\hline Li & 0.546 & 0.743 & 0.715 & 0.712 & 0.263 & -0.161 & -0.636 & 0.168 & 0.550 & 0.527 & 0.488 & -0.198 & -0.624 & -0.420 & 0.205 & 1.000 & & & & & \\
\hline Cu & 0.304 & 0.302 & 0.315 & 0.236 & 0.736 & -0.073 & -0.357 & 0.600 & 0.346 & 0.191 & 0.478 & -0.261 & -0.354 & -0.330 & 0.263 & 0.253 & 1.000 & & & & \\
\hline $\mathrm{Pb}$ & 0.359 & 0.276 & 0.285 & 0.322 & 0.186 & -0.142 & -0.359 & 0.161 & 0.288 & 0.040 & 0.315 & -0.219 & -0.366 & -0.244 & 0.349 & 0.179 & 0.029 & 1.000 & & & \\
\hline $\mathrm{Zn}$ & 0.631 & 0.832 & 0.846 & 0.907 & 0.316 & -0.223 & -0.757 & 0.363 & 0.724 & 0.501 & 0.665 & -0.187 & -0.755 & -0.545 & 0.144 & 0.691 & 0.272 & 0.269 & 1.000 & & \\
\hline Co & 0.409 & 0.635 & 0.588 & 0.528 & 0.794 & 0.097 & -0.540 & 0.474 & 0.621 & 0.489 & 0.661 & -0.298 & -0.541 & -0.386 & 0.252 & 0.377 & 0.471 & 0.223 & 0.492 & 1.000 & \\
\hline $\mathrm{Ni}$ & 0.300 & 0.517 & 0.513 & 0.418 & 0.810 & 0.048 & -0.433 & 0.563 & 0.539 & 0.552 & 0.643 & -0.188 & -0.447 & -0.310 & 0.201 & 0.295 & 0.622 & 0.097 & 0.431 & 0.829 & 1.000 \\
\hline
\end{tabular}

\section{TABLE 10}

Matrix of Correlation Coefficients for Mesozoic Sediments Analyzed from DSDP Sites 259-263 Leg 27 (when $\rho \geqslant 0.80$ and $a=0.05$, the correlation coefficient is equal to 0.680 )

\begin{tabular}{|c|c|c|c|c|c|c|c|c|c|c|c|c|c|c|c|c|c|c|c|c|c|}
\hline & $\mathrm{SiO}_{2}$ & $\mathrm{TiO}_{2}$ & $\mathrm{Al}_{2} \mathrm{O}_{3}$ & $\mathrm{Fe}_{2} \mathrm{O}_{3}$ & $\mathrm{MnO}$ & $\mathrm{MgO}$ & $\mathrm{CaO}$ & $\mathrm{Na}_{2} \mathrm{O}$ & $\mathrm{K}_{2} \mathrm{O}$ & $\mathrm{P}_{2} \mathrm{O}_{5}$ & $\mathrm{H}_{2} \mathrm{O}$ & Res. & $\mathrm{CO}_{2}$ & Sr & $\mathrm{Ba}$ & Li & $\mathrm{Cu}$ & $\mathrm{Pb}$ & $\mathrm{zn}$ & Co & $\mathrm{Ni}$ \\
\hline $\mathrm{SiO}_{2}$ & 1.000 & & & & & & & & & & & & & & & & & & & & \\
\hline $\mathrm{TiO}_{2}$ & 0.135 & 1.000 & & & & & & & & & & & & & & & & & & & \\
\hline $\mathrm{Al}_{2} \mathrm{O}_{3}$ & 0.087 & 0.853 & 1.000 & & & & & & & & & & & & & & & & & & \\
\hline $\mathrm{Fe}_{2} \mathrm{O}_{3}$ & 0.070 & 0.756 & 0.636 & 1.000 & & & & & & & & & & & & & & & & & \\
\hline $\mathrm{MnO}$ & -0.332 & 0.013 & -0.048 & 0.029 & 1.000 & & & & & & & & & & & & & & & & \\
\hline $\mathrm{MgO}$ & 0.218 & 0.680 & 0.556 & 0.464 & 0.350 & 1.000 & & & & & & & & & & & & & & & \\
\hline $\mathrm{CaO}$ & -0.861 & -0.559 & -0.549 & -0.442 & 0.214 & -0.547 & 1.000 & & & & & & & & & & & & & & \\
\hline $\mathrm{Na}_{2} \mathrm{O}$ & 0.154 & 0.231 & 0.302 & -0.035 & 0.261 & 0.594 & -0.361 & 1.000 & & & & & & & & & & & & & \\
\hline $\mathrm{K}_{2} \mathrm{O}$ & 0.236 & 0.697 & 0.644 & 0.445 & 0.245 & 0.775 & -0.592 & 0.718 & 1.000 & & & & & & & & & & & & \\
\hline $\mathrm{P}_{2} \mathrm{O}_{5}$ & -0.026 & 0.575 & 0.457 & 0.721 & 0.167 & 0.487 & -0.252 & -0.131 & 0.334 & 1.000 & & & & & & & & & & & \\
\hline $\mathrm{H}_{2} \mathrm{O}$ & 0.221 & 0.556 & 0.684 & 0.308 & 0.184 & 0.731 & -0.585 & 0.773 & 0.786 & 0.166 & 1.000 & & & & & & & & & & \\
\hline Res & -0.357 & 0.112 & 0.189 & 0.189 & -0.105 & -0.234 & 0.225 & -0.167 & -0.064 & -0.008 & -0.185 & 1.000 & & & & & & & & & \\
\hline $\mathrm{CO}_{2}$ & -0.864 & -0.538 & -0.536 & -0.393 & 0.194 & -0.557 & 0.995 & -0.399 & -0.615 & -0.212 & -0.601 & 0.208 & 1.000 & & & & & & & & \\
\hline $\mathrm{Sr}$ & -0.521 & -0.295 & -0.264 & -0.175 & 0.433 & 0.009 & 0.489 & 0.308 & 0.030 & -0.154 & -0.049 & 0.367 & 0.452 & 1.000 & & & & & & & \\
\hline $\mathrm{Ba}$ & 0.395 & -0.335 & -0.291 & -0.239 & 0.189 & 0.167 & -0.208 & 0.122 & -0.048 & 0.058 & -0.001 & -0.308 & -0.226 & 0.023 & 1.000 & & & & & & \\
\hline $\mathrm{Li}$ & 0.183 & 0.554 & 0.501 & 0.548 & -0.181 & 0.158 & -0.358 & -0.291 & 0.202 & 0.528 & -0.009 & 0.110 & -0.314 & -0.333 & -0.059 & 1.000 & & & & & \\
\hline $\mathrm{Cu}$ & -0.204 & -0.169 & -0.357 & -0.100 & 0.679 & 0.114 & 0.261 & 0.145 & 0.107 & -0.001 & -0.078 & -0.199 & 0.235 & 0.313 & 0.085 & -0.278 & 1.000 & & & & \\
\hline $\mathrm{Pb}$ & 0.170 & 0.098 & 0.101 & 0.134 & 0.207 & 0.211 & -0.186 & -0.018 & -0.005 & 0.043 & 0.067 & -0.074 & -0.188 & -0.002 & 0.297 & 0.016 & -0.091 & 1.000 & & & \\
\hline $\mathrm{Zn}$ & 0.030 & 0.648 & 0.692 & 0.817 & -0.105 & 0.361 & -0.379 & -0.048 & 0.333 & 0.593 & 0.232 & 0.361 & -0.341 & -0.109 & -0.302 & 0.496 & -0.249 & 0.059 & 1.000 & & \\
\hline Co & 0.062 & 0.465 & 0.397 & 0.290 & 0.733 & 0.703 & -0.307 & 0.416 & 0.555 & 0.346 & 0.505 & -0.203 & -0.323 & 0.124 & 0.079 & 0.052 & 0.448 & 0.184 & 0.226 & 1.000 & \\
\hline $\mathrm{Ni}$ & -0.102 & 0.290 & 0.204 & 0.181 & 0.664 & 0.621 & -0.100 & 0.486 & 0.544 & 0.353 & 0.370 & -0.042 & -0.138 & 0.387 & 0.052 & -0.194 & 0.519 & 0.029 & 0.145 & 0.793 & 1.000 \\
\hline
\end{tabular}


TABLE 11

Matrix of Correlation Coefficients for Cenozoic Sediments Analyzed from DSDP Sites 259-263 Leg 27 (when $\rho \geqslant 0.80$ and $a=0.05$, the correlation coefficient is equal to 0.598 )

\begin{tabular}{|c|c|c|c|c|c|c|c|c|c|c|c|c|c|c|c|c|c|c|c|c|c|}
\hline & $\mathrm{SiO}_{2}$ & $\mathrm{TiO}_{2}$ & $\mathrm{Al}_{2} \mathrm{O}_{3}$ & $\mathrm{Fe}_{2} \mathrm{O}_{3}$ & $\mathrm{MnO}$ & $\mathrm{MgO}$ & $\mathrm{CaO}$ & $\mathrm{Na}_{2} \mathrm{O}$ & $\mathrm{K}_{2} \mathrm{O}$ & $\mathrm{P}_{2} \mathrm{O}_{5}$ & $\mathrm{H}_{2} \mathrm{O}$ & Res & $\mathrm{CO}_{2}$ & $\mathrm{Sr}$ & $\mathrm{Ba}$ & $\mathrm{Li}$ & $\mathrm{Cu}$ & $\mathrm{Pb}$ & $\mathrm{Zn}$ & Co & $\mathrm{Ni}$ \\
\hline $\mathrm{SiO}_{2}$ & 1.000 & & & & & & & & & & & & & & & & & & & & \\
\hline $\mathrm{TiO}_{2}$ & 0.897 & 1.000 & & & & & & & & & & & & & & & & & & & \\
\hline $\mathrm{Al}_{2} \mathrm{O}_{3}$ & 0.955 & 0.979 & 1.000 & & & & & & & & & & & & & & & & & & \\
\hline $\mathrm{Fe}_{2} \mathrm{O}_{3}$ & 0.913 & 0.956 & 0.957 & 1.000 & & & & & & & & & & & & & & & & & \\
\hline $\mathrm{MnO}$ & 0.685 & 0.742 & 0.730 & 0.651 & 1.000 & & & & & & & & & & & & & & & & \\
\hline $\mathrm{MgO}$ & -0.378 & -0.274 & -0.330 & -0.275 & -0.005 & 1.000 & & & & & & & & & & & & & & & \\
\hline $\mathrm{CaO}$ & -0.978 & -0.930 & -0.969 & -0.937 & -0.754 & 0.222 & 1.000 & & & & & & & & & & & & & & \\
\hline $\mathrm{Na}_{2} \mathrm{O}$ & 0.848 & 0.688 & 0.756 & 0.665 & 0.720 & -0.439 & -0.810 & 1.000 & & & & & & & & & & & & & \\
\hline $\mathrm{K}_{2} \mathrm{O}$ & 0.940 & 0.890 & 0.929 & 0.958 & 0.585 & -0.329 & -0.941 & 0.748 & 1.000 & & & & & & & & & & & & \\
\hline $\mathrm{P}_{2} \mathrm{O}_{5}$ & 0.500 & 0.617 & 0.574 & 0.654 & 0.673 & 0.032 & 0.572 & 0.288 & 0.523 & 1.000 & & & & & & & & & & & \\
\hline $\mathrm{H}_{2} \mathrm{O}$ & 0.934 & 0.924 & 0.947 & 0.922 & 0.802 & -0.302 & -0.953 & 0.821 & 0.908 & 0.640 & 1.000 & & & & & & & & & & \\
\hline Res & -0.155 & -0.176 & -0.161 & -0.168 & -0.299 & 0.083 & 0.130 & -0.101 & -0.129 & -0.173 & -0.246 & 1.000 & & & & & & & & & \\
\hline $\mathrm{CO}_{2}$ & -0.989 & -0.935 & -0.976 & -0.952 & -0.714 & 0.341 & 0.989 & -0.818 & -0.955 & -0.580 & -0.958 & 0.114 & 1.000 & & & & & & & & \\
\hline Sr & -0.466 & -0.433 & -0.464 & -0.450 & -0.514 & -0.266 & 0.571 & -0.441 & -0.460 & -0.216 & -0.469 & -0.183 & 0.498 & 1.000 & & & & & & & \\
\hline $\mathrm{Ba}$ & 0.823 & 0.700 & 0.783 & 0.643 & 0.743 & -0.426 & -0.796 & 0.903 & 0.689 & 0.672 & 0.791 & -0.111 & -0.793 & -0.457 & 1.000 & & & & & & \\
\hline Li & 0.865 & 0.964 & 0.945 & 0.898 & 0.819 & -0.229 & -0.902 & 0.688 & 0.818 & 0.257 & 0.915 & -0.223 & -0.901 & -0.387 & 0.703 & 1.000 & & & & & \\
\hline Cu & 0.625 & 0.565 & 0.592 & 0.444 & 0.886 & -0.074 & -0.650 & 0.811 & 0.480 & 0.353 & 0.672 & -0.266 & -0.604 & -0.429 & 0.812 & 0.635 & 1.000 & & & & \\
\hline $\mathrm{Pb}$ & 0.367 & 0.264 & 0.333 & 0.332 & 0.063 & -0.238 & -0.342 & 0.312 & 0.403 & 0.061 & 0.435 & -0.183 & -0.362 & -0.141 & 0.230 & 0.252 & 0.062 & 1.000 & & & \\
\hline $\mathrm{Zn}$ & 0.948 & 0.940 & 0.964 & 0.964 & 0.728 & -0.339 & -0.955 & 0.775 & 0.944 & 0.612 & 0.954 & -0.219 & -0.968 & -0.472 & 0.769 & 0.899 & 0.590 & 0.339 & 1.000 & & \\
\hline Co & 0.644 & 0.806 & 0.748 & 0.763 & 0.896 & 0.037 & -0.741 & 0.527 & 0.640 & 0.857 & 0.813 & -0.231 & -0.720 & -0.492 & 0.527 & 0.852 & 0.623 & 0.101 & 0.763 & 1.000 & \\
\hline$\underline{\mathrm{Ni}}$ & 0.676 & 0.793 & 0.759 & 0.734 & 0.932 & -0.040 & -0.753 & 0.608 & 0.614 & 0.818 & 0.827 & -0.220 & -0.735 & -0.473 & 0.625 & 0.869 & 0.684 & 0.126 & 0.763 & 0.972 & 1.000 \\
\hline
\end{tabular}

TABLE 12

Matrix of Correlation Coefficients for Clays from DSDP Sites 259-263 Leg 27 (when $\rho \geqslant 0.80$ and $a=0.05$, the correlation coefficient is equal to 0.609)

\begin{tabular}{|c|c|c|c|c|c|c|c|c|c|c|c|c|c|c|c|c|c|c|c|c|c|}
\hline & $\mathrm{SiO}_{2}$ & $\mathrm{TiO}_{2}$ & $\mathrm{Al}_{2} \mathrm{O}_{3}$ & $\mathrm{Fe}_{2} \mathrm{O}_{3}$ & $\mathrm{MnO}$ & $\mathrm{MgO}$ & $\mathrm{CaO}$ & $\mathrm{Na}_{2} \mathrm{O}$ & $\mathrm{K}_{2} \mathrm{O}$ & $\mathrm{P}_{2} \mathrm{O}_{5}$ & $\mathrm{H}_{2} \mathrm{O}$ & Res & $\mathrm{CO}_{2}$ & Sr & $\mathrm{Ba}$ & Li & $\mathrm{Cu}$ & $\mathrm{Pb}$ & $\mathrm{Zn}$ & Co & $\mathrm{Ni}$ \\
\hline $\mathrm{SiO}_{2}$ & 1.000 & & & & & & & & & & & & & & & & & & & & \\
\hline $\mathrm{TiO}_{2}$ & -0.710 & 1.000 & & & & & & & & & & & & & & & & & & & \\
\hline $\mathrm{Al}_{2} \mathrm{O}_{3}$ & -0.873 & 0.800 & 1.000 & & & & & & & & & & & & & & & & & & \\
\hline $\mathrm{Fe}_{2} \mathrm{O}_{3}$ & -0.642 & 0.730 & 0.585 & 1.000 & & & & & & & & & & & & & & & & & \\
\hline $\mathrm{MnO}$ & -0.388 & 0.208 & 0.271 & 0.063 & 1.000 & & & & & & & & & & & & & & & & \\
\hline $\mathrm{MgO}$ & -0.585 & 0.582 & 0.498 & 0.300 & 0.632 & 1.000 & & & & & & & & & & & & & & & \\
\hline $\mathrm{CaO}$ & -0.378 & -0.155 & 0.053 & 0.067 & -0.115 & -0.128 & 1.000 & & & & & & & & & & & & & & \\
\hline $\mathrm{Na}_{2} \mathrm{O}$ & -0.336 & 0.071 & 0.202 & -0.274 & 0.565 & 0.414 & -0.011 & 1.000 & & & & & & & & & & & & & \\
\hline $\mathrm{K}_{2} \mathrm{O}$ & -0.630 & 0.593 & 0.499 & 0.277 & 0.353 & 0.566 & 0.128 & 0.505 & 1.000 & & & & & & & & & & & & \\
\hline $\mathrm{P}_{2} \mathrm{O}_{5}$ & -0.554 & 0.585 & 0.531 & 0.628 & 0.391 & 0.612 & -0.036 & -0.103 & 0.230 & 1.000 & & & & & & & & & & & \\
\hline $\mathrm{H}_{2} \mathrm{O}$ & -0.669 & 0.391 & 0.583 & 0.099 & 0.617 & 0.718 & -0.047 & 0.717 & 0.581 & 0.304 & 1.000 & & & & & & & & & & \\
\hline Res & -0.373 & 0.417 & 0.432 & 0.509 & -0.141 & -0.048 & 0.146 & -0.299 & 0.113 & 0.176 & -0.125 & 1.000 & & & & & & & & & \\
\hline $\mathrm{CO}_{2}$ & -0.334 & -0.104 & 0.051 & 0.262 & -0.158 & -0.261 & 0.843 & -0.076 & -0.085 & 0.056 & -0.166 & 0.035 & 1.000 & & & & & & & & \\
\hline Sr & -0.452 & 0.144 & 0.196 & 0.052 & 0.602 & 0.459 & 0.282 & 0.620 & 0.559 & 0.002 & 0.491 & 0.019 & 0.087 & 1.000 & & & & & & & \\
\hline $\mathrm{Ba}$ & 0,444 & -0.535 & -0.482 & -0.414 & 0.175 & -0.008 & -0.169 & 0.066 & -0.244 & -0.054 & -0.141 & -0.356 & -0.202 & 0.091 & 1.000 & & & & & & \\
\hline $\mathrm{Li}$ & -0.284 & 0.481 & 0.439 & 0.471 & 0.069 & 0.121 & -0.076 & -0.322 & 0.004 & 0.545 & -0.099 & 0.271 & 0.129 & -0.235 & -0.148 & 1.000 & & & & & \\
\hline $\mathrm{Cu}$ & -0.177 & 0.148 & 0.155 & -0.192 & 0.730 & 0.289 & -0.180 & 0.685 & 0.092 & 0.134 & 0.471 & -0.222 & -0.056 & 0.259 & 0.100 & 0.081 & 1.000 & & & & \\
\hline $\mathrm{Pb}$ & 0.092 & -0.129 & -0.062 & 0.077 & $0.097^{\circ}$ & 0.043 & -0.052 & -0.151 & -0.135 & -0.158 & -0.132 & -0.048 & -0.064 & 0.165 & 0.279 & -0.088 & -0.136 & 1.000 & & & \\
\hline $\mathrm{Zn}$ & -0.652 & 0.616 & 0.705 & 0.769 & 0.054 & 0.236 & 0.137 & -0.157 & 0.153 & 0.529 & 0.106 & 0.625 & 0.286 & 0.069 & -0.460 & 0.446 & -0.029 & 0.030 & 1.000 & & \\
\hline Co & -0.419 & 0.435 & 0.408 & 0.203 & 0.810 & 0.75 ? & -0.232 & 0.367 & 0.471 & 0.448 & 0.570 & -0.082 & -0.346 & 0.549 & -0.012 & 0.064 & 0.381 & 0.073 & 0.173 & 1.000 & \\
\hline $\mathrm{Ni}$ & -0.481 & 0.364 & 0.400 & 0.096 & 0.815 & 0.734 & -0.075 & 0.489 & 0.390 & 0.556 & 0.648 & -0.024 & -0.227 & 0.451 & -0.038 & 0.045 & 0.564 & -0.092 & 0.151 & 0.815 & 1.000 \\
\hline
\end{tabular}




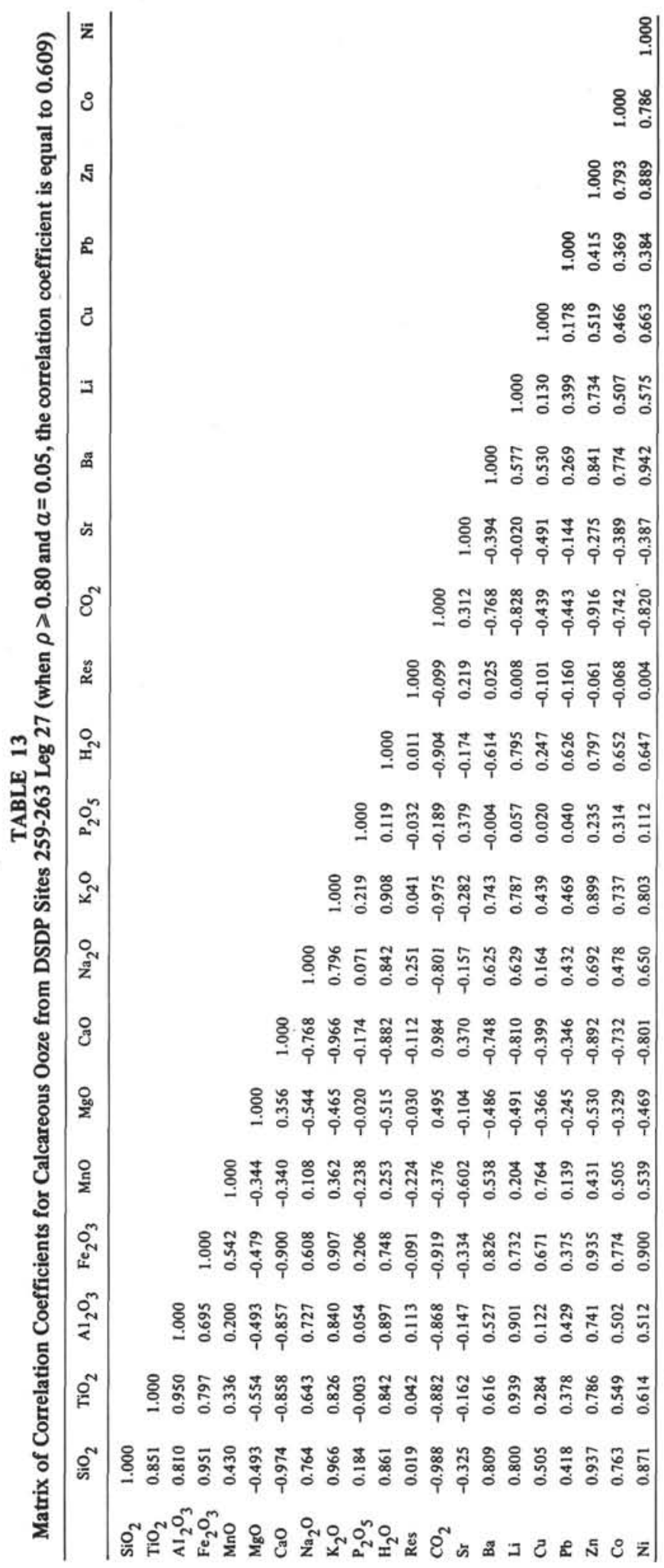

The vertical variation of factor scores in a calcareous ooze sequence at Site 262 is shown in Figure 8. Various trends are discernible, though few are likely to be of any real geologic significance. The increase of factor scores in Factor 1 is merely a reflection of increasing carbonate with depth. Factor 5 (MgO-Sr) results from increasing dolomite with increasing depth. There are no definite factor score trends in Factors 3, 4, or 6.

Turning now to the results of R-mode factor analysis on the clays, which are primarily of Mesozoic age, it is apparent that the factor groupings are rather different from those obtained for calcareous ooze. The six factors explain $84 \%$ of the total variance. Factor 1 (Figure 7) is responsible for $34 \%$ of the total variance and consequently would appear to be an important factor, but it is composed of a puzzling group of variables including $\mathrm{MnO}, \mathrm{Na}_{2} \mathrm{O}, \mathrm{Cu}$, and $\mathrm{Ni}$. It is possible that this factor is in part a function of elemental scavenging by manganese oxides, but there is no obvious reason for the inclusion of $\mathrm{Na}_{2} \mathrm{O}$ with this factor.

Factor 2 is probably a biogenic silica-aluminosilicate factor. Influx of biogenic silica results in dilution of other variables, particularly clay minerals. The association of $\mathrm{SiO}_{2}$ and $\mathrm{Ba}$ in Factor 2 is important in that it supports the suggestion of Brongersma-Sanders (1966) that much of the $\mathrm{Ba}$ is associated with biogenic silica (such as that from diatoms) rather than with volcanic sources, as Bostrom et al. (1973) have suggested.

Factor 3, which is comprised primarily of $\mathrm{CaO}$ and $\mathrm{CO}_{2}$ and accounts for $11 \%$ of the total variance, is fairly certainly controlled by the influx of biogenic calcium carbonate. Factor 4 is considerably more complex and includes a large number of variables. As in Factor 2, the negative factor loading for $\mathrm{SiO}_{2}$ points to the dilution effect of biogenic silica. Other controlling mechanisms would'seem to be aluminosilicates (accounting for the variables $\mathrm{TiO}_{2}, \mathrm{Al}_{2} \mathrm{O}_{3}, \mathrm{MgO}$ [?]), $\mathrm{Na}_{2} \mathrm{O}, \mathrm{K}_{2} \mathrm{O}$, and $\mathrm{H}_{2} \mathrm{O}$ ) and manganese oxides probably occurring as small nodules and grain coatings (accounting for the variables $\mathrm{MnO}, \mathrm{Co}$, and $\mathrm{Ni}$ ). The reason for the occurrence of $\mathrm{Sr}$ in Factor 4 is not obvious though it may be associated in part with $\mathrm{MgO}$, as in the case in Factor 5 for calcareous ooze (Figure 6).

Factor 5 accounts for changes in lead and barium values; this is again a puzzling combination of variables. It does suggest that although much of the barium may be associated with biogenic silica, some is associated with base metals and may be concentrated by elemental scavenging either by clays or by oxides. Factor 6 does not appear to have any geologic significance.

The vertical variation in factor scores for clays at Site 263 is shown in Figure 9. Factor 1 shows a fairly constant vertical variation, though it is evident that the two samples (55 and 56, see Figure 2) at the bottom of the hole are somewhat atypical. This is not entirely unexpected as these samples contain abundant kaolinite and detrital quartz. If Samples 55 and 56 are excluded from consideration in Factor 2, there is a marked increase in the factor score with increasing depth, which is probably a reflection of the decreasing importance of biogenic silica with increasing depth. 


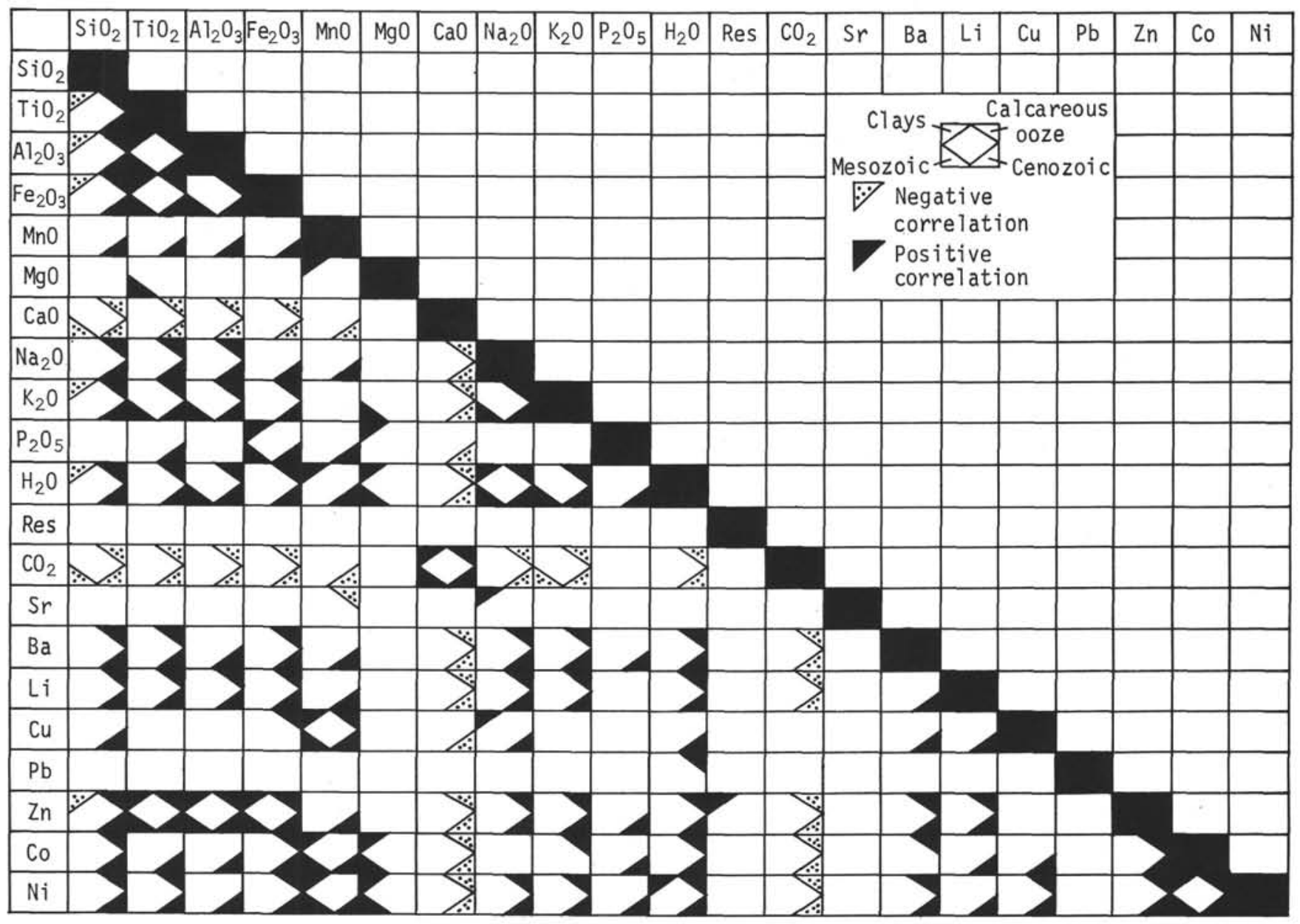

Figure 5. Matrix of correlation coefficients for the clay-calcareous ooze and Mesozoic-Cenozoic groupings.

FACTOR LOADINGS

CALCAREOUS 002

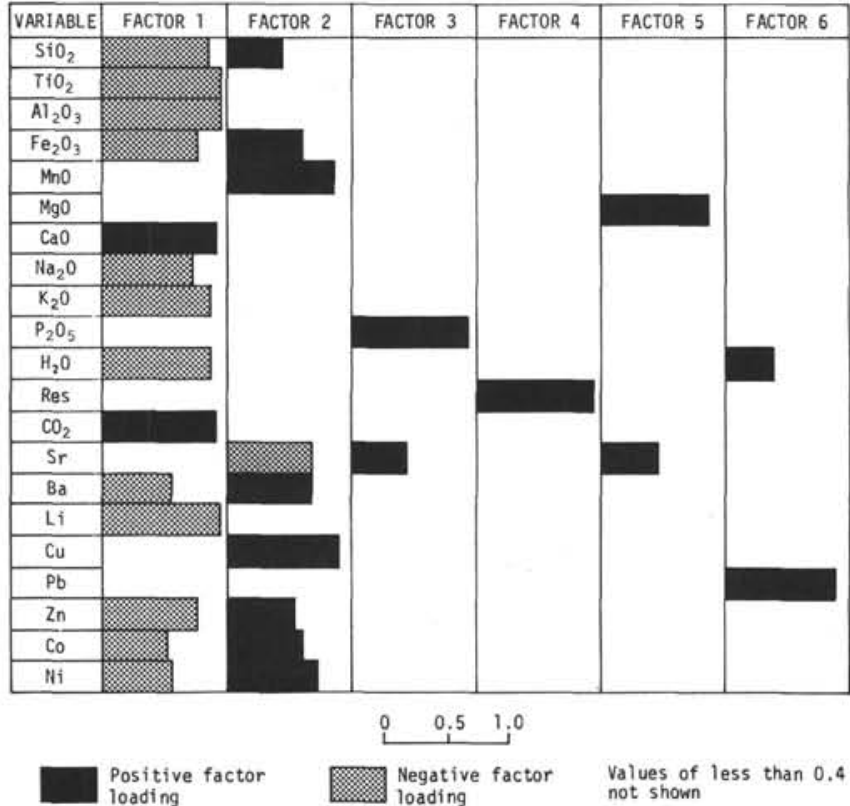

Figure 6. Graphical representation of Varimax rotated factor loadings for calcareous ooze.

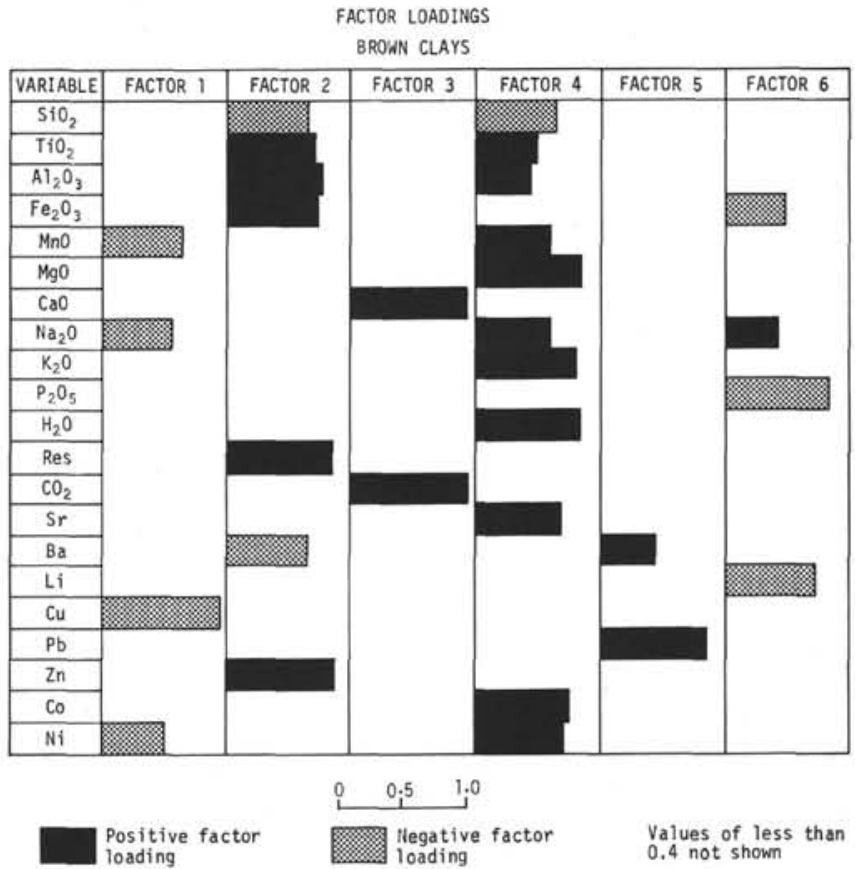

Figure 7. Graphical representation of Varimax rotated factor loadings for clay. 


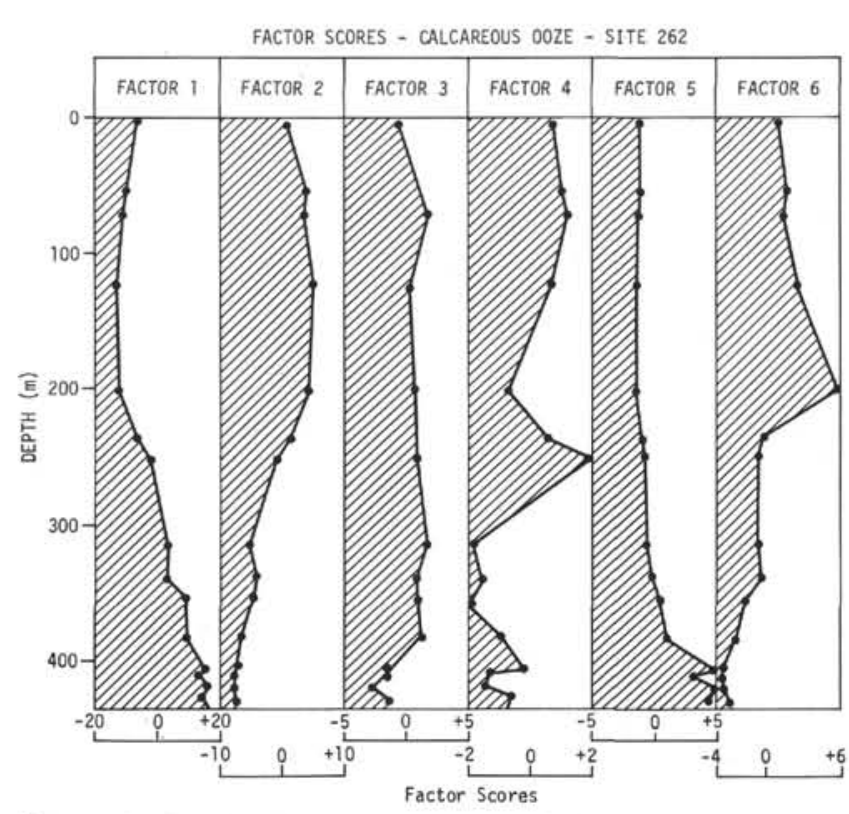

Figure 8. Graphical representation of the vertical variation of factor scores in Cenozoic calcareous ooze at Site 262.

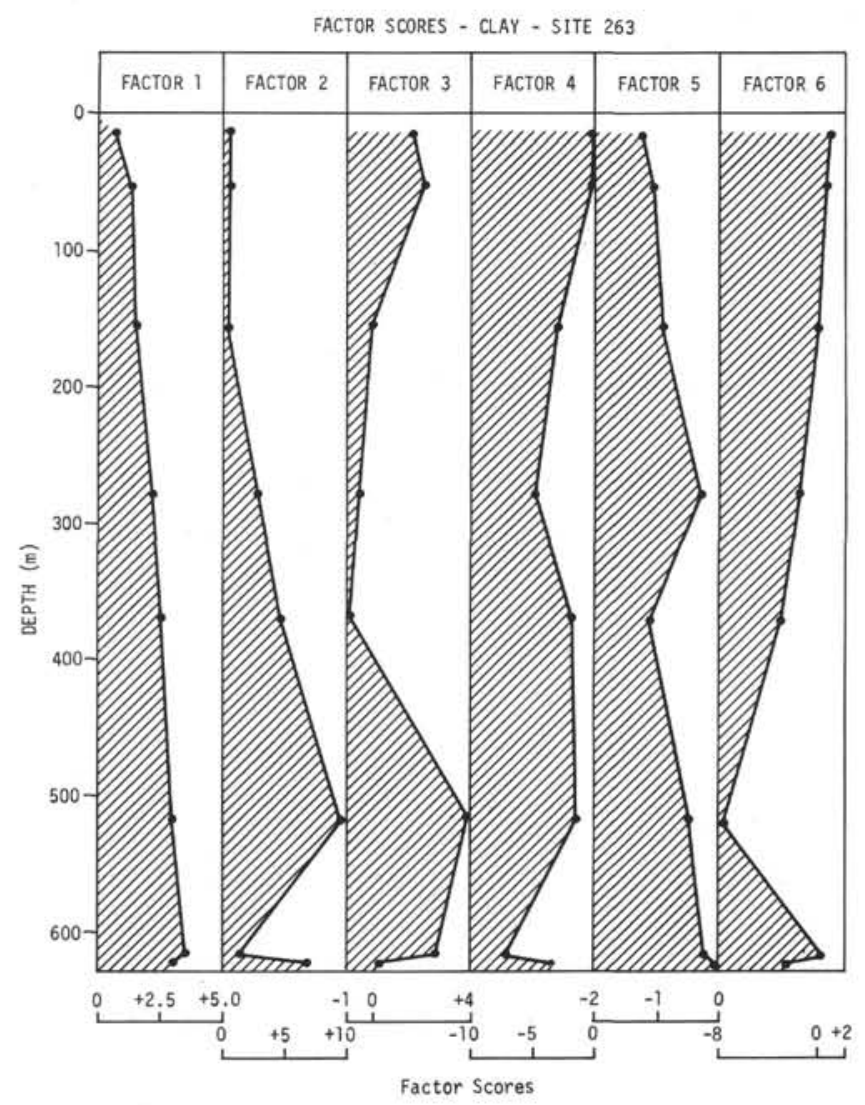

Figure 9. Graphical representation of the vertical variation of factor scores in Mesozoic clay at Site 263.
It is not possible to attach any geologic significance to any of the other factor score trends in Figure 9.

\section{CONCLUSIONS}

1. Sediments obtained on Leg 27 of the Deep Sea Drilling Project fall into two main types-clay which is mainly Mesozoic in age and calcareous ooze which is mainly Cenozoic in age.

2. If considered on a carbonate-free, water-free basis, these two groups of sediments are similar to each other and to pelagic sediments from elsewhere.

3. The Indian Ocean sediments obtained from Leg 27 are somewhat impoverished in trace elements when compared with global oceanic averages.

4. Multivariate analysis was of only limited usefulness in the study of Leg 27 sediments because of the problem of the dilution effect of $\mathrm{CaCO}_{3}$ coupled with the result of the closed array effect (constant summation to $100 \%$ of major elements).

5 . Oxides and elements may be combined by the use of correlation coefficients and R-mode factor analysis into one or more groups including: (1) the aluminosilicate group- $\mathrm{SiO}_{2}, \mathrm{Al}_{2} \mathrm{O}_{3}, \mathrm{TiO}_{2}, \mathrm{Fe}_{2} \mathrm{O}_{3}$, $\mathrm{Na} 2 \mathrm{O}, \mathrm{K}_{2} \mathrm{O}, \mathrm{H}_{2} \mathrm{O}, \mathrm{Zn}$; (b) the ferromanganese group- $\mathrm{Fe}_{2} \mathrm{O}_{3}, \mathrm{MnO}, \mathrm{Cu}, \mathrm{Zn}, \mathrm{Co}, \mathrm{Ni}$; (c) the biogenic carbonate group- $\mathrm{CaO}, \mathrm{CO}_{2}, \mathrm{Sr}($ ?); and (d) the biogenic silica group- $\mathrm{SiO}_{2}, \mathrm{Ba}$.

6. There is the suggestion of $\mathrm{Fe}_{2} \mathrm{O}_{3}-\mathrm{P}_{2} \mathrm{O}_{5}$ factor which may be a reflection of adsorption of $\mathrm{P}_{2} \mathrm{O}_{5}$ by oxides, or, alternatively, is a response to the formation of trace amounts of vivianite.

7. A number of variables are notable for their lack of meaningful associations, including $\mathrm{MgO}, \mathrm{Li}$, and $\mathrm{Pb}$.

\section{ACKNOWLEDGMENTS}

The cooperation of Mr. A. B. Timms and the Analytical Chemistry Section of the Australian Mineral Development Laboratories was most helpful. Advice on all aspects of the statistical treatment of the data was freely given by $\mathrm{Mr}$. W. Mayo of the Bureau of Mineral Resources, Canberra.

\section{REFERENCES}

Bostrom, K., Joensuu, O., Moore, C., Bostrom, B., Dalziel, M., and Horowliz, A., 1973. Geochemistry of barium in pelagic sediments: Lithos, v. 6, p. 159-174.

Bowditch, D. C., Cowan, A., and Lomman, R. F., 1969. Rock analysis by direct reading emission spectroscopy: Australian Inst. Mining Metal. Proc., v. 229, p. 121-128.

Brogersma-Sanders, M., 1966. Barium in pelagic sediments and diatoms: Konikl. Ned. Akad. Wetensch. Proc., v. 70, p. 93-99.

Chave, K. E. and Mackenzie, F. T., 1961. A statistical technique applied to the geochemistry of pelagic muds: J. Geol., v. 69, p. 572-582.

Chayes, F., 1960. On correlation between variables of constant sum: J. Geophys. Res., v. 65, p. 4185-4193.

Dixon, W. J. (Ed.), 1971. Biomedical computer programs: Berkeley (Univ. Calif. Press).

El Wakeel, S. K. and Riley, J. P., 1961. Chemical and mineralogical studies of deep sea sediments: Geochim. Cosmochim. Acta, v. 25, p. 110-146.

Goldberg, E. D. and Arrhenius, G., 1958. Chemistry of Pacific pelagic sediments: Geochim. Cosmochim. Acta, v. 13, p. 153-212. 
Hirst, D. M. and Kaye, M. J., 1971. Factors controlling the mineralogy and chemistry over an upper Visean sedimentary sequence from Rookhope, County Durham: Chem. Geol., v. 8, p. 37-59.

Krauskopf, K. R., 1956. Factors controlling the concentration of thirteen rare metals in seawater: Geochim. Cosmochim. Acta, v. 9, p. 1-32.
Olson, E. C. and Miller, R. L., 1958. Morphological integration: Chicago, (University Press).

Summerhayes, C. P., 1972. Geochemistry of continental margin sediments from northwest Africa: Chem. Geol., v. 10 , p. $137-157$

Turekian, K. K. and Wedepohl, K. H., 1961. Distribution of the elements in some major rock units in the earth's crust: Geol. Soc. Am. Bull., v. 172, p. 175-192. 